\title{
Emergence of quasiperiodicity in symmetrically coupled, identical period-doubling systems
}

Reick, Christian; Mosekilde, Erik

Published in:

Physical Review E. Statistical, Nonlinear, and Soft Matter Physics

Link to article, DOI:

10.1103/PhysRevE.52.1418

Publication date:

1995

Document Version

Publisher's PDF, also known as Version of record

Link back to DTU Orbit

Citation (APA):

Reick, C., \& Mosekilde, E. (1995). Emergence of quasiperiodicity in symmetrically coupled, identical perioddoubling systems. Physical Review E. Statistical, Nonlinear, and Soft Matter Physics, 52(2), 1418-1435. https://doi.org/10.1103/PhysRevE.52.1418

\section{General rights}

Copyright and moral rights for the publications made accessible in the public portal are retained by the authors and/or other copyright owners and it is a condition of accessing publications that users recognise and abide by the legal requirements associated with these rights.

- Users may download and print one copy of any publication from the public portal for the purpose of private study or research.

- You may not further distribute the material or use it for any profit-making activity or commercial gain

- You may freely distribute the URL identifying the publication in the public portal 


\title{
Emergence of quasiperiodicity in symmetrically coupled, identical period-doubling systems
}

\author{
Christian Reick \\ I. Institut für Theoretische Physik, Universität Hamburg, D-20355 Hamburg, Germany \\ Erik Mosekilde \\ Physics Department, Center for Chaos and Turbulence Studies, The Technical University of Denmark, DK-2800 Lyngby, Denmark
}

(Received 13 December 1994)

\begin{abstract}
When two identical period-doubling systems are coupled symmetrically, the period-doubling transition to chaos may be replaced by a quasiperiodic transition. The reason for this is that at an early stage of the period-doubling cascade, a Hopf bifurcation instead of a period-doubling bifurcation occurs. Our main result is that the emergence of this Hopf bifurcation is a generic phenomenon in symmetrically coupled, identical period-doubling systems. The whole phenomenon is stable against small nonsymmetric perturbations. Our results cover maps and differential equations of arbitrary dimension. As a consequence the Feigenbaum transition to chaos in these coupled systems - which exists, but tends to be unstable-is accompanied by an infinity of Hopf bifurcations.
\end{abstract}

PACS number(s): 05.45. $+\mathrm{b}, 02.90 .+\mathrm{p}$

\section{INTRODUCTION}

When two oscillators with different frequencies interact, the dynamics is typically quasiperiodic for sufficiently small coupling strength and may undergo a quasiperiodic transition to chaos when the nonlinearity is increased [1]. A similar transition is found, when two identical period-doubling systems are coupled symmetrically, although the uncoupled systems now oscillate at the same frequency. This phenomenon has been observed experimentally by Van Buskirk and Jeffries [2]. They investigated driven passive resonators composed of an inductance in series with a $p-n$ junction acting as a nonlinear capacitance. When varying the drive voltage the single resonator showed a period-doubling transition to chaos, whereas two resonators coupled resistively exhibited a quasiperiodic transition. The same phenomenon was reported by Anishchenko [3], who investigated a system of two inductively coupled electronic frequency generators.

Both Van Buskirk and Jeffries, and Anishchenko, noted that a replacement of a period-doubling transition by a quasiperiodic transition also takes place if one couples a logistic map symmetrically to another identical logistic map. Systems of two identical, symmetrically coupled one-dimensional (1D) maps have been studied in detail by Frøyland [4], in order to clarify the typical behavior of these systems. He also found this change from a perioddoubling to a quasiperiodic transition. The same phenomenon was also noted by Paulus, Gass, and Mandell [5] for a pair of multiplicatively coupled maps. Based on a comparison of the numerically obtained bifurcations in coupled logistic maps and coupled sine maps, Hogg and Hubermann [6] claimed that the bifurcations in these systems are generic for coupled oscillators.

Apparently no general explanation for this replacement of a period-doubling transition by a quasiperiodic transition has been given so far. In this paper we describe in detail the bifurcations leading to quasiperiodicity that arise when two identical period-doubling systems are coupled symmetrically. Here symmetric coupling means that the coupled system is invariant under the exchange $x \leftrightarrow y$, where $x$ and $y$ are the state variables of the two single systems. We show that the emergence of quasiperiodicity in these systems is generic. These quasiperiodic solutions need not be stable. In this case they do not show up experimentally and the coupled system behaves for weak coupling as the uncoupled system.

Nevertheless there is, as we show, a tendency for the replacement of the period-doubling transition in the single system by a quasiperiodic transition in the coupled system. Therefore this change in the type of transition to chaos may be observed in various coupled perioddoubling systems. It may show up, for instance, in twocell reactor experiments of the Belousov-Zhabotinsky reaction $[7,8]$ or the peroxidase-oxidase reaction, because the single-cell reactor is known to undergo a perioddoubling transition to chaos [9-11]. The change in the transition may also be observed in the dynamics of railway wheel sets. In a model for a single wheel set a period-doubling transition is found [12]. For waggons with two wheel sets, these are coupled, and a quasiperiodic transition may take place. Another example could be the dripping faucet experiment [13]. The single faucet shows a period-doubling transition and two dripping faucets, interacting, for instance, via mechanical vibrations transmitted from one to the other as a drop falls, may undergo a quasiperiodic transition. A period-doubling transition has also been found in a model for isolated nephrons [14]. Nephrons, the functional elements of the kidney, interact via a variety of different mechanisms, and with this coupling they may show a quasiperiodic transition instead of a period-doubling transition. Finally, we expect that this change in the type of transition may be 
found in numerous coupled electronic oscillators, where the single oscillator undergoes a period-doubling transition [15].

Before stating in more detail what we prove in this paper, we illustrate in Figs. 1, 2, and 3 how this change from a period-doubling to a quasiperiodic transition occurs in three different pairs of symmetrically coupled, identical period-doubling systems. The systems are coupled logistic maps (Fig. 1), coupled Henon-type maps (Fig. 2), and a pair of coupled Rössler systems (Fig. 3). In all cases figure (a) shows the bifurcation diagram of the single system. Numerically, these figures were obtained by following the dynamics for a given value of the control parameter $\lambda$ over a long time and then taking the last point as the initial value for the dynamics at a slightly larger value for $\lambda$. The figures denoted by (b) were obtained in the same way, but now with a second identical system coupled symmetrically to the first. The result is that the cascade of period doublings is interrupted and instead of the usual period-doubling pitchforks now filled pitchforks appear. What typically happens inside these filled pitchforks is shown in Fig. 4. Here we plotted for the two coupled logistic maps simultaneously their amplitudes for several values of the control parameter $\lambda$. For values of $\lambda$ close to the branching point of the filled pitchfork, the motion covers a closed curve and is quasiperiodic. For larger values of $\lambda$ the curves get bumpy andinterrupted by periodic windows (not visible in Fig. 4) -
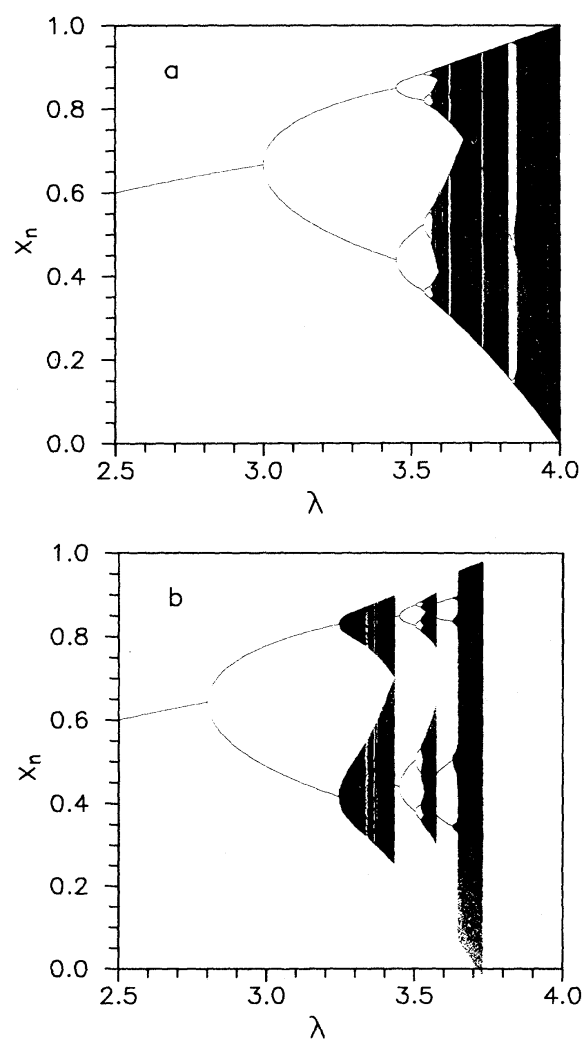

FIG. 1. Symmetrically coupled logistic maps; see Eqs. (2.1) and (2.2). (a) Uncoupled: $\epsilon=0$; (b) coupled: $\epsilon=0$.1. Beyond $\lambda \approx 3.72$ our algorithm failed to find an attractor. a quasiperiodic transition to chaos takes place.

The clue to this change from the period-doubling transition to the quasiperiodic transition is the change of a period-doubling bifurcation into a Hopf bifurcation not present in the single system. Due to this Hopf bifurcation the dynamics obtains access to the additional degrees of freedom introduced by the coupling that finally allows for the quasiperiodic transition to chaos instead of the period-doubling transition. Therefore our study focuses on the mechanism leading to this Hopf bifurcation.

The general bifurcation scheme leading to this Hopf bifurcation is sketched in Fig. 5. Figure 5(a) illustrates how the single period-doubling system behaves. The fixed point first undergoes a bifurcation that produces a periodic solution. In the case of maps this is a perioddoubling bifurcation and in the case of differential equations a Hopf bifurcation. Then the periodic solution becomes unstable by (another) period-doubling bifurcation. In the coupled system, depending on the sign of the coupling, two different situations are possible. The first, realized in the examples shown in Figs. 1-3, is sketched in Fig. 5(b). Here the first bifurcation of the symmetric fixed point produces a periodic solution with a broken symmetry. This nonsymmetric solution then undergoes the Hopf bifurcation we are interested in. It turns out
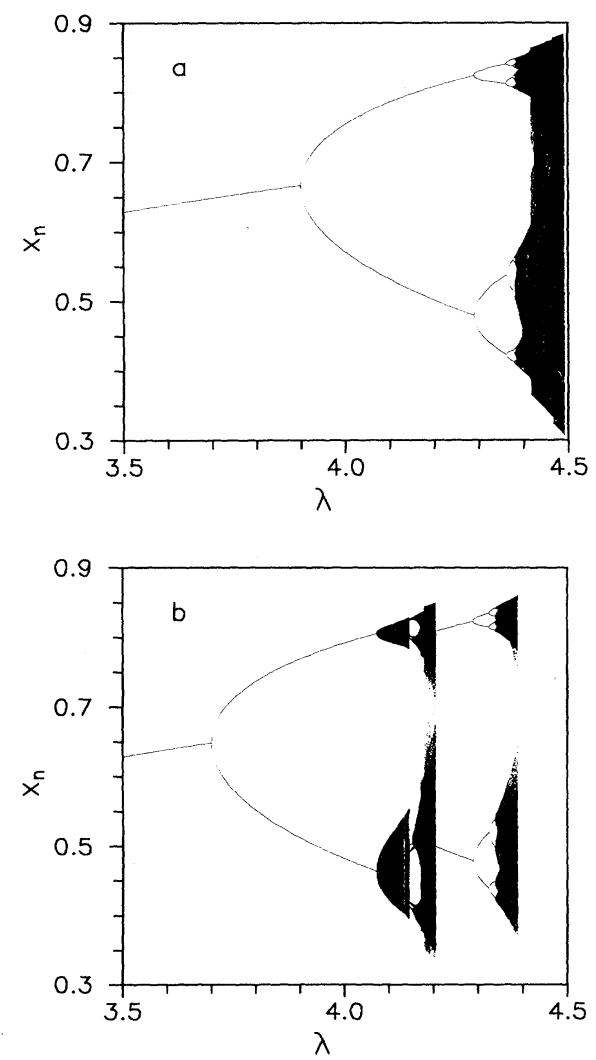

FIG. 2. Symmetrically coupled Henon-type maps: $x_{n+1}=f_{\lambda}\left(x_{n}\right)+y_{n}+\epsilon\left(p_{n}-x_{n}\right), \quad y_{n+1}=-b x_{n}, \quad p_{n+1}=f_{\lambda}\left(p_{n}\right)$ $+q_{n}+\epsilon\left(x_{n}-p_{n}\right), q_{n+1}=-b p_{n}$, where $f_{\lambda}$ is the logistic map Eq. (2.2). $b=0.3$. (a) Uncoupled: $\epsilon=0$; (b) coupled: $\epsilon=0.1$. Beyond $\lambda \approx 4.39$ our algorithm failed to find an attractor. 
that the nonsymmetric solution has the particular feature that the two subsystems oscillate $180^{\circ}$ out of phase. The original symmetric fixed point still exists after the symmetry breaking bifurcation, but is unstable. It bifurcates into a symmetric periodic solution. So the bifurcation of the fixed point in the single system gives rise to two bifurcations in the coupled system. The second situation is sketched in Fig. 5(c). Here the symmetry breaking and symmetry conserving bifurcations of the fixed point appear in reversed order. In such a case, the procedure used to compute Figs. 1-3 would show a period doubling of the symmetric solution instead of the Hopf bifurcation of the now unstable nonsymmetric solution. Nevertheless, the Feigenbaum route may then be interrupted at higher bifurcations, because the same bifurcations we so far discussed only for a fixed point are possible for periodic solutions as well. In Fig. 1(b) we see this for two coupled logistic maps around $\lambda=3.5$, where a symmetric orbit of period 4 doubles into a nonsymmetric orbit of period 8 . Here the period- 8 orbit undergoes the Hopf bifurcation.

We show in the following that for sufficiently weak coupling strength $\epsilon$, depending on the sign of $\epsilon$, one of the bifurcation schemes Fig. 5(b) or Fig. 5(c) is generically valid for pairs of symmetrically coupled, identical maps
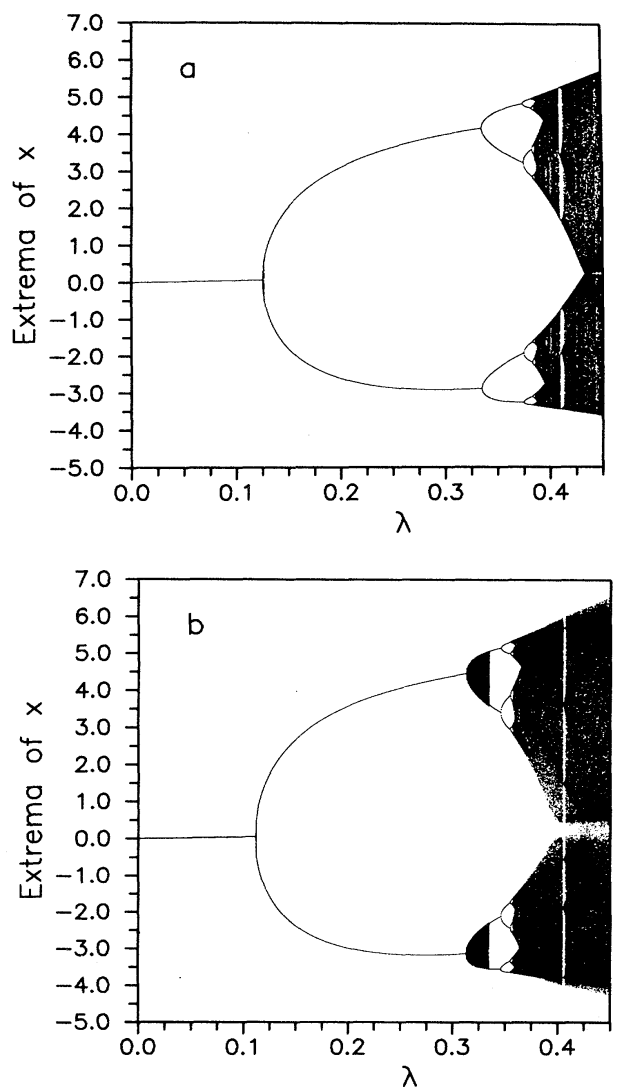

FIG. 3. Symmetrically coupled Rössler systems: $\dot{x}=-(y+z), \quad \dot{y}=x+\lambda y, \quad \dot{z}=b+z(x-c)+\epsilon(w-z), \quad \dot{u}=-(v$ $+w), \dot{v}=u+\lambda v, \dot{w}=b+w(u-c)+\epsilon(z-w)$ with parameters $b=2$ and $c=4$. Plotted are subsequent extrema of $x(t)$. (a) Uncoupled system: $\epsilon=0$; (b) coupled system: $\epsilon=0.25$.

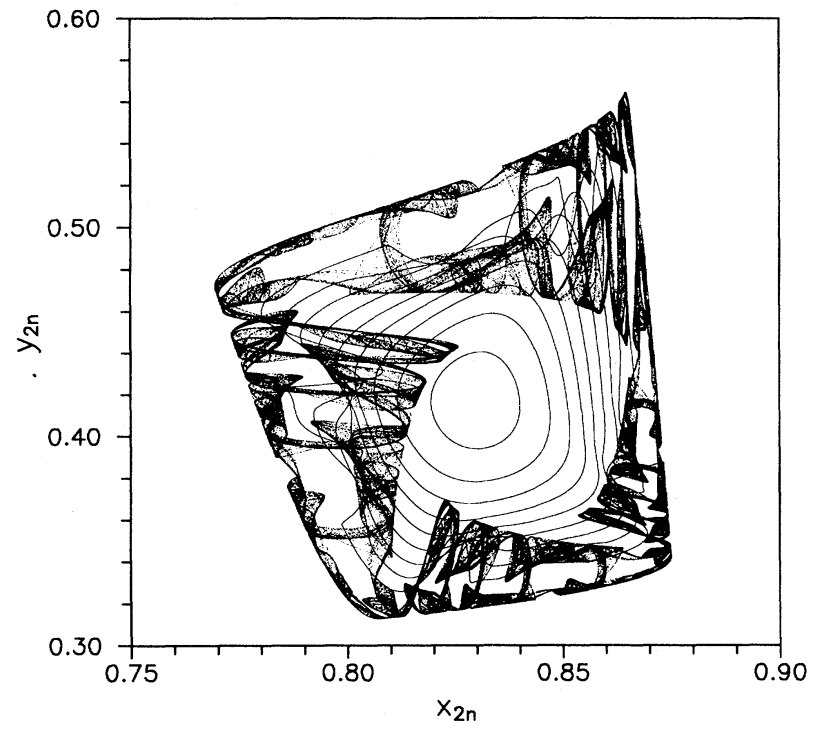

FIG. 4. Quasiperiodicity and chaos in the coupled logistic maps [Eqs. (2.1) and (2.2)] for $\epsilon=0.1$ fixed. Following the Hopf bifurcation of the antiphase orbit at $\lambda \approx 3.245$ quasiperiodic solutions, frequency locking, and chaos appear. Shown are nine quasiperiodic solutions for $\lambda=3.25,3.26, \ldots, 3.33$. Here, larger $\lambda$ corresponds to larger radius. At $\lambda=3.34$ a frequencylocked solution with period 42 appears (not visible). The wild structure is a chaotic orbit with Lyapunov exponent $L \approx 0.04$ for $\lambda=3.35$.

$$
\begin{aligned}
& x_{n+1}=f_{\lambda}\left(x_{n}\right)+\epsilon g_{\epsilon}\left(x_{n}, y_{n}\right), \\
& y_{n+1}=f_{\lambda}\left(y_{n}\right)+\epsilon g_{\epsilon}\left(y_{n}, x_{n}\right)
\end{aligned}
$$

$\left(x_{n}, y_{n} \in \mathbb{R}^{N}\right)$ and symmetrically coupled, identical systems of differential equations

$$
\begin{aligned}
& \dot{x}=F_{\lambda}(x)+\epsilon G_{\epsilon}(x, y), \\
& \dot{y}=F_{\lambda}(y)+\epsilon G_{\epsilon}(y, x)
\end{aligned}
$$

$\left(x, y \in \mathbb{R}^{N}\right)$, supposing for the single systems $x_{n+1}=f_{\lambda}\left(x_{n}\right)$ and $\dot{x}=F_{\lambda}(x)$ the bifurcation diagram Fig. 5(a) holds. We also show that these results remain valid in the presence of weak symmetry breaking terms.

As shown in Fig. 5, the first bifurcation in the single system splits into two bifurcations in the coupled system. This splitting has been extensively studied for symmetrically coupled, identical systems of differential equations, where each single system is close to a Hopf bifurcation. The seemingly first remark on this splitting is due to Ruelle [16]. He noted that the symmetry of the coupling results in a splitting into a symmetric solution and a nonsymmetric solution, where for the latter the two subsystems are $180^{\circ}$ out of phase. Later Neu [17] specialized the problem to linear coupling and analyzed the stability of the symmetric and nonsymmetric solutions by singular perturbation methods. Using symmetry arguments Klič [18] showed that a period-doubling bifurcation of the nonsymmetric solution is nongeneric. Kawato and Suzuki [19] realized in a special case that the solution re- 

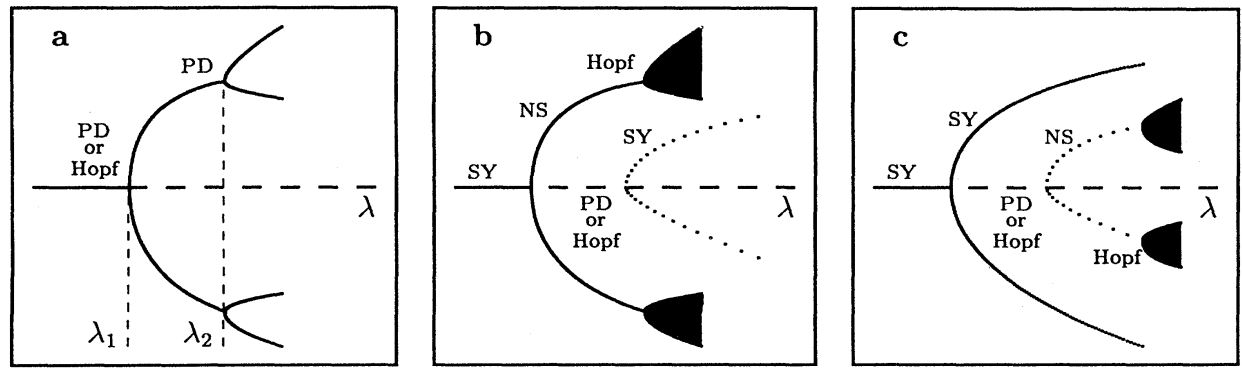

FIG. 5. Schematic bifurcation diagrams. (a) Bifurcations in the single system, or, equivalently, in the uncoupled system. (b) and (c) Bifurcations in the symmetrically coupled system. Abbreviations: $\mathbf{P D}=$ period-doubling bifurcation, $S Y=$ symmetric solution, NS= nonsymmetric solution. sulting from the second Hopf bifurcation, which is usually unstable, recovers stability by a secondary bifurcation $[20,21]$. They argue that this is a general phenomenon in symmetrically coupled, identical oscillators. Preliminary results indicate that these secondary bifurcations indeed show up in our three examples, but we will not consider them in the following.

For this case, where both $N$-dimensional subsystems are close to a Hopf bifurcation, the center manifold of the weakly coupled system is four dimensional, because the center manifold of the single-system Hopf bifurcation is two dimensional. Therefore the whole $2 N$-dimensional problem can be reduced to the study of a pair of coupled two-dimensional systems close to a Hopf bifurcation. Many studies of this situation exist [22-26]. But the situation we consider here is different: In our case the single system undergoes two subsequent bifurcations, where the second is a period-doubling bifurcation, and this perioddoubling bifurcation is only possible if the single system of differential equations is at least three dimensional. Therefore studies of coupled two-dimensional systems do not lead to the phenomenon of the emergence of quasiperiodicity we analyze here.

The paper is organized as follows. In Sec. II the example of two symmetrically coupled logistic maps is discussed in detail in order to understand the relevant features of the problem. In Sec. III we prove our claims for coupled maps and in Sec. IV for systems of coupled differential equations. This will be done by a perturbation expansion in the coupling strength $\epsilon$. Section IV depends on Sec. III, because we repeatedly take over results from Sec. III. In the Appendix we prove two propositions that are needed in Secs. III and IV.

\section{TWO SYMMETRICALLY COUPLED LOGISTIC MAPS}

The emergence of quasiperiodic behavior in coupled period-doubling systems can be studied in detail in the following system of two symmetrically coupled logistic maps [3,4,6,27-29]:

$$
\begin{aligned}
& x_{n+1}=f_{\lambda}\left(x_{n}\right)+\epsilon\left(y_{n}-x_{n}\right), \\
& y_{n+1}=f_{\lambda}\left(y_{n}\right)+\epsilon\left(x_{n}-y_{n}\right),
\end{aligned}
$$

with

$$
f_{\lambda}(z)=\lambda z(1-z),
$$

where $\lambda$ is the bifurcation parameter of the logistic map $f$ and $\epsilon$ the coupling strength. In the following we explicit- ly show that, depending on the sign of $\epsilon$, one of the bifurcation diagrams Figs. 5(b) or 5(c) is valid.

The coupled system (2.1) is invariant under the symmetry operation

$$
\mathcal{\rho}\left(\begin{array}{l}
x \\
y
\end{array}\right) \equiv\left(\begin{array}{l}
y \\
x
\end{array}\right)
$$

Because of this symmetry the system has either symmetric or nonsymmetric solutions. The special coupling in (2.1) vanishes for $x_{n}=y_{n}$ so that the symmetric solutions of (2.1) are related to the solutions $z_{n}$ of the logistic map (2.2) by

$$
\left[\begin{array}{l}
x_{n}^{(s)} \\
y_{n}^{(s)}
\end{array}\right)=z_{n}\left[\begin{array}{l}
1 \\
1
\end{array}\right] \text { with } z_{n+1}=f_{\lambda}\left(z_{n}\right) .
$$

Therefore all symmetric orbits of the coupled system can be inferred from solutions of the logistic map.

From (2.1) and (2.2) one obtains that the symmetric fixed point of the coupled system is given by

$$
\left[\begin{array}{l}
x^{(s)} \\
y^{(s)}
\end{array}\right]=z\left[\begin{array}{l}
1 \\
1
\end{array}\right] \text { with } z=f_{\lambda}(z)=\frac{\lambda-1}{\lambda} .
$$

A symmetric period-2 orbit reads

$q_{n}^{(s)} \equiv\left(\begin{array}{l}x_{n}^{(s)} \\ y_{n}^{(s)}\end{array}\right)=z_{n}\left(\begin{array}{l}1 \\ 1\end{array}\right)$ with $z_{n+1}=f_{\lambda}\left(z_{n}\right)=a+b(-1)^{n}$,

where

$$
a=\frac{\lambda+1}{2 \lambda} \text { and } b^{2}=\frac{(\lambda-1)^{2}-4}{4 \lambda^{2}} .
$$

But although symmetric orbits of the coupled system (2.1) follow from orbits of the logistic map, they may have different stability. If $\left(x_{n+p}^{(s)}, y_{n+p}^{(s)}\right)=\left(x_{n}^{(s)}, y_{n}^{(s)}\right)$ is a period- $p$ orbit of the coupled system, then its stability is determined by the eigenvalues of the matrix

$$
J=\prod_{n=1}^{p}\left[\begin{array}{cc}
f_{\lambda}^{\prime}\left(x_{n}^{(s)}\right)-\epsilon & \epsilon \\
\epsilon & f_{\lambda}^{\prime}\left(y_{n}^{(s)}\right)-\epsilon
\end{array}\right)
$$

For a symmetric orbit $\left(z_{n+p}, z_{n+p}\right)=\left(z_{n}, z_{n}\right)$ the stability matrix $J$ has the eigenvalues [30,31]

$$
\begin{aligned}
& \eta_{1}=\prod_{n=1}^{p} f_{\lambda}^{\prime}\left(z_{n}\right), \\
& \eta_{2}=\prod_{n=1}^{p}\left(f_{\lambda}^{\prime}\left(z_{n}\right)-2 \epsilon\right) .
\end{aligned}
$$


The first of these two eigenvalues is equal to the stability coefficient

$$
\gamma \equiv \prod_{n=1}^{p} f_{\lambda}^{\prime}\left(z_{n}\right)
$$

of the period- $p$ orbit $(z)_{1, \ldots, p}$ of the logistic map itself. A periodic orbit of the coupled system is stable if and only if $\left|\eta_{1}\right|$ and $\left|\eta_{2}\right|$ are smaller than 1 , but the stability of the orbit $(z)_{1, \ldots, p}$ of the logistic map guarantees only $\left|\eta_{1}\right|<1$. Whether $\left|\eta_{2}\right|$ is smaller than 1 depends also on the coupling strength $\epsilon$. For the symmetric fixed point (2.5) one finds

$$
\begin{aligned}
& \eta_{1}=2-\lambda, \\
& \eta_{2}=2-\lambda-2 \epsilon,
\end{aligned}
$$

and for symmetric period-2 orbit (2.6) and (2.7)

$$
\begin{aligned}
& \eta_{1}=5-(\lambda-1)^{2}, \\
& \eta_{2}=5-(\lambda-1)^{2}+4 \epsilon(\epsilon+1) .
\end{aligned}
$$

Because the eigenvalue $\eta_{1}$ of the stability matrix $J$ of symmetric solutions is equal to the stability coefficient $\gamma$ of the logistic map, period-doubling bifurcations of symmetric orbits appear at the same parameter values as in the logistic map and thus produce a new symmetric orbit. Therefore at $\lambda_{1}=3$ the symmetric fixed point (2.5) undergoes a period-doubling bifurcation to the symmetric orbit (2.6) and (2.7), which itself doubles into a symmetric period-4 orbit at $\lambda_{2}=1+\sqrt{6}$. Nevertheless, the symmetric orbits produced in these bifurcations need not be stable. Whether they are stable or not depends on the sign of $\epsilon$. For example, for positive $\epsilon$ the eigenvalue $\eta_{2}$ of the symmetric fixed point becomes -1 at $\lambda=3-2 \epsilon$ before $\eta_{1}$ becomes -1 . So here another period-doubling bifurcation occurs, not present in the logistic map.

This period-doubling bifurcation produces the nonsymmetric period-2 orbit

$q_{n}^{(n)} \equiv\left[\begin{array}{l}x_{n}^{(n)} \\ y_{n}^{(n)}\end{array}\right]=\left[\begin{array}{c}z_{n}^{(n)} \\ z_{n+1}^{(n)}\end{array}\right] \quad$ with $z_{n}^{(n)}=c+d(-1)^{n}$

where

$$
c=\frac{\lambda+1-2 \epsilon}{2 \lambda}, \quad d^{2}=\frac{(\lambda-1)^{2}-4(\epsilon-1)^{2}}{4 \lambda^{2}} .
$$

This period- 2 orbit has the interesting property that the iteration $n \rightarrow n+1$ is equal to the symmetry operation

$$
q_{1}^{(n)}=S q_{0}^{(n)}, \quad q_{0}^{(n)}=S q_{1}^{(n)} .
$$

Solutions with this property have been called antiphase solutions by Marek and Schreiber [32,33]. (In the literature other names also such as "out-of-phase" solution [6], "incoherent" solution [4], or simply "asymmetric" solution [3] are in use.) Correspondingly the symmetric solution is sometimes called an in-phase solution. The antiphase property (2.15) is a direct consequence of the symmetry of the system. For $\epsilon \neq 0$ each period-doubling bifurcation of the symmetric fixed point produces exactly one period-2 solution $\left(q_{n}\right)_{1,2}$. Because the system con- sidered is invariant under $\mathcal{S}$ the orbit $\left(\mathscr{S} q_{n}\right)_{1,2}$ has also to be a solution. There are only two possibilities: (a) $\mathcal{S} q_{n}=q_{n}$, i.e., the solution is symmetric, or (b) $\mathcal{S} q_{0}=q_{1}$, $\mathcal{S} q_{1}=q_{0}$, i.e., the solution has the antiphase property (2.15). This reasoning holds not only for coupled logistic maps, but for symmetric maps in general and has a counterpart for coupled systems of differential equations with symmetry.

The eigenvalues of the stability matrix $J$ of the period-2 antiphase orbit (2.13) and (2.14) are

$$
\begin{aligned}
\eta_{1,2}= & 5(\epsilon-1)^{2}-(\lambda-1)^{2}+\epsilon^{2} \\
& \pm 2 \epsilon \sqrt{5(\epsilon-1)^{2}-(\lambda-1)^{2}} .
\end{aligned}
$$

For $\lambda>1+\sqrt{5}(1-\epsilon)$ these two eigenvalues are complex conjugate and leave the unit circle at

$$
\lambda_{H}=1+\sqrt{2(1-\epsilon)(3-2 \epsilon)} .
$$

Hence the antiphase orbit undergoes a Hopf bifurcation and for almost all $\epsilon$, quasiperiodicity emerges (compare Fig. 4). This completes the analysis of the first bifurcations. It shows that-depending on the sign of $\epsilon$-one of the bifurcation diagrams Fig. 5(b) or 5(c) applies.

With regard to the following sections it is interesting to consider the behavior of the system (2.1) in the weakcoupling limit $\epsilon \rightarrow 0$. For $\epsilon=0$, i.e., when the system is uncoupled, the in-phase and antiphase solutions are given by

$$
q_{0}^{(s)}=\left(\begin{array}{l}
z_{0} \\
z_{0}
\end{array}\right), \quad q_{1}^{(s)}=\left(\begin{array}{l}
z_{1} \\
z_{1}
\end{array}\right)
$$

and

$$
q_{0}^{(n)}=\left(\begin{array}{l}
z_{0} \\
z_{1}
\end{array}\right), \quad q_{1}^{(n)}=\left(\begin{array}{l}
z_{1} \\
z_{0}
\end{array}\right),
$$

respectively, with $z_{0}=f_{\lambda}\left(z_{1}\right)$ and $z_{1}=f_{\lambda}\left(z_{0}\right)$. So the inphase and anti-phase solutions of the coupled system emerge from the in-phase and anti-phase solutions of the uncoupled system that may be constructed from the possible combinations of the single-system period-2 solutions $z_{0}, z_{1}$. This remark is not restricted to coupled logistic maps, but is generally true for coupled identical systems. Moreover, this explains why the two period-doubling bifurcations of the symmetric fixed point (2.5) produce an in-phase and an antiphase solution, and not only solutions of one type: Already the uncoupled system possesses an in-phase and an antiphase solution. These remarks are of special importance for an understanding of our procedure in the next section, where we expand the antiphase solution of the coupled system up to order $\epsilon$ in the antiphase solution of the uncoupled system in order to prove that the antiphase solution undergoes a Hopf bifurcation for $|\epsilon|$ sufficiently small but nonzero.

In continuing the analysis of the weak-coupling limit we expand the eigenvalues (2.16) of the stability matrix $J$ of the antiphase orbit in $\epsilon$. This gives

$$
\eta_{1,2}=\gamma-2 \epsilon(5 \pm \sqrt{\gamma})+O\left(\epsilon^{2}\right),
$$

where $\gamma=5-(\lambda-1)^{2}$ is the stability coefficient of the 
period-2 orbit $z_{0}, z_{1}$ of the logistic map. For $\epsilon=0,(2.20)$ reduces to $\eta_{1,2}=\gamma$ and the antiphase orbit of the uncoupled system undergoes a period-doubling transition when $\gamma$ crosses -1 . This is in accordance with the known single-system behavior. For $\epsilon \neq 0$ there is an additional imaginary contribution to $\eta_{1,2}$ because $\sqrt{\gamma}$ is imaginary for $\gamma$ close to -1 . So close to the period-2-period-4 bifurcation of the logistic map the coupled system has two complex conjugate eigenvalues. Moreover, for sufficiently small $\epsilon$, Eq. (2.20) shows that it is always possible to change $\lambda$ so that $\left|\eta_{1,2}\right|$ gets larger than 1 , because by changing $\lambda$ we can sweep $|\gamma|$ through 1 . This illustrates once more-here in order $\epsilon$ - that the antiphase solution undergoes a Hopf bifurcation. Moreover, the discussion shows that the emergence of this Hopf bifurcation is intimately connected with the period-2-period-4 bifurcation in the single system. This type of behavior will be recovered in the general cases of symmetrically coupled systems. Specifically, an equation with the same structure as (2.20), which relates the stability of the antiphase solution to the stability of the period-2 solution of the single system, will be recovered.

\section{TWO IDENTICAL, SYMMETRICALLY COUPLED MAPS}

In this section we consider the following general system of two identical, symmetrically coupled perioddoubling maps:

$$
\begin{aligned}
& x_{n+1}=f_{\lambda}\left(x_{n}\right)+\epsilon g_{\epsilon}\left(x_{n}, y_{n}\right), \\
& y_{n+1}=f_{\lambda}\left(y_{n}\right)+\epsilon h_{\epsilon}\left(x_{n}, y_{n}\right),
\end{aligned}
$$

with

$$
h_{\epsilon}(x, y)=g_{\epsilon}(y, x)
$$

guaranteeing the symmetry $x \leftrightarrow y, x, y \in \mathbb{R}^{N}$ and $g_{\epsilon}$ an analytic function of the coupling parameter $\epsilon$. The single system

$$
z_{n+1}=f_{\lambda}\left(z_{n}\right), \quad z_{n} \in \mathbb{R}^{N}
$$

is assumed to have a fixed point $z$ that undergoes a period-doubling bifurcation at the control-parameter value $\lambda=\lambda_{1}$ resulting in a period- 2 orbit $z_{0}, z_{1}$ that becomes unstable in another period-doubling bifurcation at $\lambda=\lambda_{2}>\lambda_{1}$. Under these conditions it will be shown that, depending on the sign of $\epsilon$, one of the bifurcation diagrams shown in Figs. 5(b) and 5(c) is generic for the coupled system. Deviations from this behavior are possible but nongeneric.

To prove the validity of the bifurcation diagrams $5(\mathrm{~b})$ and 5(c), one has to show two things: First that the fixed point in the coupled system undergoes two perioddoubling bifurcations, one leading to an in-phase orbit and the other to an antiphase orbit; and second that the antiphase orbit undergoes a Hopf bifurcation. These proofs are the content of the first two subsections III A and III B. In the remaining two subsections III C and III $D$ the results will be extended, first to higher-order period doublings (Sec. III C) and then to systems where the symmetry is broken by additional terms on the right hand side of (3.1) (Sec. III D).

\section{A. Period-doubling bifurcations of the fixed point}

From the assumed properties of the single system it follows immediately that the uncoupled system

$$
\left(x_{n+1}, y_{n+1}\right)=\left(f_{\lambda}\left(x_{n}\right), f_{\lambda}\left(y_{n}\right)\right)
$$

has for $\lambda<\lambda_{1}$ a symmetric fixed point $(z, z)$. At $\lambda_{1}$ this fixed point undergoes a degenerate period-doubling bifurcation resulting in a symmetric in-phase orbit $\left(z_{0}, z_{0}\right),\left(z_{1}, z_{1}\right)$ and an antiphase orbit $\left(z_{0}, z_{1}\right),\left(z_{1}, z_{0}\right)$. In this period-doubling bifurcation two eigenvalues of the stability matrix of the fixed point $(z, z)$ pass through -1 . If now the coupling is turned on these orbits persist but the two eigenvalues depend on $\epsilon$ and thus - in the generic case-differ. It will be shown below that for sufficiently weak coupling the two eigenvalues are still real valued. Therefore the coupling does not change the type of bifurcation: both eigenvalues pass through -1 but now at different parameter values $\lambda$. Moreover, the coupled system is still symmetric, which implies that its period- 2 orbits bifurcating from a symmetric fixed point have to be in-phase or antiphase orbits (compare Sec. II). Also, because the type of an orbit cannot change abruptly in the limit $\epsilon \rightarrow 0$, in-phase and antiphase orbits in the coupled system relate to in-phase and antiphase orbits in the uncoupled system. So, because there are already an in-phase and an antiphase orbit in the uncoupled system, one of the two period doublings in the coupled system has to produce an in-phase orbit from the fixed point and the other an antiphase orbit. Hence the first part of the bifurcation diagrams 5 (b) or 5 (c) correctly describes the situation in the coupled system.

It remains to be shown that the eigenvalues of the stability matrix of the fixed point remain real for small coupling. The alternative would be a pair of complexconjugate eigenvalues. But this alternative can be excluded as follows. Let $(\bar{z}, \bar{z})$ denote the symmetric fixed point of the coupled system. For the stability matrix at this fixed point one obtains from (3.1)

$$
\begin{aligned}
J & \left.\equiv \frac{\partial\left(x_{n+1}, y_{n+1}\right)}{\partial\left(x_{n}, y_{n}\right)}\right|_{(\bar{z}, \bar{z})} \\
& =\left[\begin{array}{cc}
\left.\frac{\partial f_{\lambda}}{\partial x}\right|_{\bar{z}} & 0 \\
0 & \left.\frac{\partial f_{\lambda}}{\partial y}\right|_{\bar{z}}
\end{array}|+\epsilon| \begin{array}{c|c|}
\left.\frac{\partial g_{\epsilon}}{\partial x}\right|_{(\bar{z}, \bar{z})} & \left.\frac{\partial g_{\epsilon}}{\partial y}\right|_{(\bar{z}, \bar{z})} \\
\left.\frac{\partial h_{\epsilon}}{\partial x}\right|_{(\bar{z}, \bar{z})} & \left.\frac{\partial h_{\epsilon}}{\partial y}\right|_{(\bar{z}, \bar{z})}
\end{array}\right) .
\end{aligned}
$$

The system (3.1) is symmetric under the symmetry operation

$$
\mathcal{S}\left(\begin{array}{l}
x \\
y
\end{array}\right)=\left(\begin{array}{l}
y \\
x
\end{array}\right) .
$$

Therefore, and because $(\bar{z}, \bar{z})$ is a symmetric fixed point, 
the stability matrix $J$ obeys the symmetry relation

$$
\mathcal{S} \mathcal{S}=J,
$$

where the representation

$$
\rho \equiv\left(\begin{array}{ll}
0 & 1 \\
1 & 0
\end{array}\right)
$$

of $\mathcal{S}$ has been used. Here $\mathbb{1}$ is the $N \times N$ unit matrix. Expanding the fixed point $(\bar{z}, \bar{z})$ of the coupled system at the fixed point $(z, z)$ of the uncoupled system in orders of $\epsilon$ gives

$$
(\bar{z}, \bar{z})=(z, z)+\epsilon(u, u)+O\left(\epsilon^{2}\right) .
$$

This expansion conserves the symmetry and thus all terms of an expansion of $J$ in orders of $\epsilon$ obey the same symmetry relation (3.6) as $J$ itself. One finds

$$
J=U+\epsilon V+O\left(\epsilon^{2}\right)
$$

with

$$
U=\left(\begin{array}{ll}
\Gamma & 0 \\
0 & \Gamma
\end{array}\right] \text { and } V=\left[\begin{array}{cc}
A & B \\
B & A
\end{array}\right),
$$

where $A$ and $B$ are $N \times N$ matrices whose details are without interest in the present context, and

$$
\Gamma=\left.\frac{\partial f_{\lambda}}{\partial x}\right|_{z}
$$

is the stability matrix of the single system at the fixed point $z$. In order to compute the eigenvalues of $J$ up to order $\epsilon$ we now diagonalize $U$ by a coordinate transformation $K$ so that Proposition 1 from the Appendix applies. By this proposition we will be able to reduce our $2 N$-dimensional problem to a two-dimensional problem. Because we are interested in the generic behavior, we can assume that $\Gamma$ is diagonalizable. So if $K_{0}$ diagonalizes $\Gamma$,

$$
K_{0}^{-1} \Gamma K_{0}=\left(\begin{array}{ccc}
\gamma_{1} & & 0 \\
& \ddots & \\
0 & & \gamma_{N}
\end{array}\right) \equiv \Gamma^{\prime},
$$

then $K$ can be chosen as

$$
K=\left[\begin{array}{cc}
K_{0} & 0 \\
0 & K_{0}
\end{array}\right] \text {. }
$$

Thus one obtains

$$
J^{\prime}=K^{-1} J K=U^{\prime}+\epsilon V^{\prime}+O\left(\epsilon^{2}\right)
$$

with

$$
U^{\prime}=\left(\begin{array}{cc}
\Gamma^{\prime} & 0 \\
0 & \Gamma^{\prime}
\end{array}\right] \text { and } V^{\prime}=\left(\begin{array}{cc}
A^{\prime} & B^{\prime} \\
B^{\prime} & A^{\prime}
\end{array}\right),
$$

where $A^{\prime}=K_{0}^{-1} A K_{0}$ and $B^{\prime}=K_{0}^{-1} B K_{0}$.

Without loss of generality it is now assumed that $\gamma_{1}$ is the eigenvalue of the single-system stability matrix $\Gamma$ that passes -1 at $\lambda_{1}$ and produces the period doubling. This eigenvalue turns up twice in $U^{\prime}$ : at the positions $\left(U^{\prime}\right)_{1,1}$ and $\left(U^{\prime}\right)_{N+1, N+1}$. Hence, from Proposition 1 in the Appendix one gets for the eigenvalues $\eta_{1,2}$ of the coupled system

$$
\eta_{1,2}=\gamma_{1}+\epsilon \delta_{1,2}+O\left(\epsilon^{2}\right),
$$

where $\delta_{1,2}$ are the eigenvalues of the $2 \times 2$ submatrix

$$
\begin{aligned}
V_{\text {sub }}^{\prime} & \equiv\left[\begin{array}{cc}
\left(V^{\prime}\right)_{1,1} & \left(V^{\prime}\right)_{1, N+1} \\
\left(V^{\prime}\right)_{N+1,1} & \left(V^{\prime}\right)_{N+1, N+1}
\end{array}\right] \\
& =\left[\begin{array}{ll}
\left(A^{\prime}\right)_{1,1} & \left(B^{\prime}\right)_{1,1} \\
\left(B^{\prime}\right)_{1,1} & \left(A^{\prime}\right)_{1,1}
\end{array}\right]
\end{aligned}
$$

of $V^{\prime}$. Since $V_{\text {sub }}^{\prime}$ is Hermitian its eigenvalues $\delta_{1,2}$ are real so that up to order $\epsilon$ the eigenvalues $\eta_{1,2}$ of $J$ are real. This holds true also for higher orders in $\epsilon$ : In the generic case $\left(B^{\prime}\right)_{1,1} \neq 0$ so that $\eta_{1}$ and $\eta_{2}$ differ in order $\epsilon$. Taking into consideration possible imaginary contributions in higher orders in $\epsilon$, this result means that the real part of $\eta_{1}$ and $\eta_{2}$ already differs in order $\epsilon$. Because $J$ is a real matrix its eigenvalues $\eta_{1,2}$ are real or complex conjugate. If they were complex conjugate their real parts would have to be identical in every order $\epsilon$. But this contradicts our previous result for order $\epsilon$. So $\eta_{1}$ and $\eta_{2}$ are real to all orders in $\epsilon$.

\section{B. The Hopf bifurcation of the antiphase orbit}

In this section our main result that the antiphase orbit generically undergoes a Hopf bifurcation is proved. The proof will be performed by expanding the antiphase orbit of the coupled system in $\epsilon$ around the antiphase orbit of the uncoupled system and we will subsequently discuss the stability in $\epsilon$. Higher orders in $\epsilon$ do not change the picture. The discussion reveals that the stability matrix has for $\lambda$ close to $\lambda_{2}$ a pair of complex eigenvalues that leave the unit circle when $\lambda$ is changed appropriately. In the generic case (no resonances) this implies a Hopf bifurcation.

The Jacobian matrices at the two points $\left(\bar{z}_{0}, \bar{z}_{1}\right)$ and $\left(\bar{z}_{1}, \bar{z}_{0}\right)$ of the antiphase orbit are given by

$$
\left.J_{0} \equiv \frac{\partial\left(x_{n+1}, y_{n+1}\right)}{\partial\left(x_{n}, y_{n}\right)}\right|_{\bar{z}_{0}, \bar{z}_{1}}
$$

and

$$
\left.J_{1} \equiv \frac{\partial\left(x_{n+1}, y_{n+1}\right)}{\partial\left(x_{n}, y_{n}\right)}\right|_{\bar{z}_{1}, \bar{z}_{0}} .
$$

The stability matrix $J$ of the antiphase orbit is related to $J_{0}$ and $J_{1}$ by

$$
J=J_{1} J_{0}
$$

To discuss the eigenvalues of $J$ we first express $J$ in terms of $J_{0}$ and $\mathcal{S}$ instead of $J_{0}$ and $J_{1}$. Let $q_{n} \equiv\left(x_{n}, y_{n}\right)$. With this condensed notation the coupled system (3.1) can be rewritten as

$$
q_{n+1}=\mathscr{F}\left(q_{n}\right)
$$

and because of the symmetry of (3.1) the function $\mathcal{F}$ obeys 


$$
\rho \mathscr{F}(\rho q)=\mathscr{F}(q),
$$

where the matrix $\mathcal{S}$ was defined in (3.7). In this notation one has for $J_{1}$

$$
\begin{aligned}
J_{1}=\left.\frac{\partial q_{n+1}}{\partial q_{n}}\right|_{\left(\bar{z}_{1}, \bar{z}_{0}\right)} & =\left.\frac{\partial \mathcal{S F}\left(\mathcal{S} q_{n}\right)}{\partial q_{n}}\right|_{\left(\bar{z}_{1}, \bar{z}_{0}\right)} \\
& =\left.\mathcal{S} \frac{\partial q_{n+1}}{\partial q_{n}}\right|_{\mathcal{S}\left(\bar{z}_{1}, \bar{z}_{0}\right)} \mathcal{S},
\end{aligned}
$$

where the last step results from an application of the chain rule and the fact that $\mathcal{S}$ is a constant matrix [compare (3.7)]. Expressing $J_{0}$ also in this condensed notation and recalling the antiphase property $\mathcal{S}\left(\bar{z}_{1}, \bar{z}_{0}\right)=\left(\bar{z}_{0}, \bar{z}_{1}\right)$ one obtains

$$
J_{1}=\rho J_{0} \rho \text {. }
$$

Inserting this into (3.18) gives

$$
J=\mathcal{S} J_{0} \mathcal{S} J_{0} \text {. }
$$

So $J$ is completely determined by $J_{0}$ and $\mathcal{S}$. As we show in the following, (3.23) implies a special structure of $J$ that will turn out to be an important precondition for the appearance of the Hopf bifurcation.

From (3.1) one obtains

$$
\begin{aligned}
& J_{0}= {\left[\begin{array}{c|c}
\left.\frac{\partial f_{\lambda}}{\partial x}\right|_{\bar{z}_{0}} & 0 \\
0 & \left.\frac{\partial f_{\lambda}}{\partial y}\right|_{\bar{z}_{1}}
\end{array}\right] } \\
&\left.+\epsilon \mid \begin{array}{c|c|}
\left.\frac{\partial g_{\epsilon}}{\partial x}\right|_{\left(\bar{z}_{0}, \bar{z}_{1}\right)} & \left.\frac{\partial g_{\epsilon}}{\partial y}\right|_{\left(\bar{z}_{0}, \bar{z}_{1}\right)} \\
\left.\frac{\partial h_{\epsilon}}{\partial x}\right|_{\left(\bar{z}_{0}, \bar{z}_{1}\right)} & \left.\frac{\partial h_{\epsilon}}{\partial y}\right|_{\left(\bar{z}_{0}, \bar{z}_{1}\right)}
\end{array}\right] .
\end{aligned}
$$

This is so far exact. Now the point $\left(\bar{z}_{0}, \bar{z}_{1}\right)$ of the antiphase orbit of the coupled system is expanded in $\epsilon$ around the respective point $\left(z_{0}, z_{1}\right)$ of the antiphase orbit of the uncoupled system:

$$
\left[\begin{array}{l}
\bar{z}_{0} \\
\bar{z}_{1}
\end{array}\right)=\left[\begin{array}{l}
z_{0} \\
z_{1}
\end{array}\right]+\epsilon\left(\begin{array}{l}
u \\
v
\end{array}\right]+O\left(\epsilon^{2}\right) .
$$

Entering this expression into $J_{0}$ gives

$$
J_{0}=\left(\begin{array}{cc}
\Gamma_{0} & 0 \\
0 & \Gamma_{1}
\end{array}\right]+\epsilon\left[\begin{array}{ll}
A & B \\
C & D
\end{array}\right]+O\left(\epsilon^{2}\right),
$$

where the explicit expressions for the $N \times N$ matrices $A$, $B, C$, and $D$ are not needed in the following and

$$
\left.\Gamma_{0} \equiv \frac{\partial f_{\lambda}}{\partial x}\right|_{z_{0}} \text { and }\left.\Gamma_{1} \equiv \frac{\partial f_{\lambda}}{\partial x}\right|_{z_{1}}
$$

are the Jacobian matrices of the single-system period-2 orbit. With this expression for $J_{0}$ one thus obtains from (3.23)

$$
J=U+\epsilon V+O\left(\epsilon^{2}\right)
$$

with

$$
U=\left(\begin{array}{cc}
\Gamma_{1} \Gamma_{0} & 0 \\
0 & \Gamma_{0} \Gamma_{1}
\end{array}\right)
$$

and

$$
V=\left(\begin{array}{cc}
\Gamma_{1} A+D \Gamma_{0} & \Gamma_{1} B+C \Gamma_{1} \\
B \Gamma_{0}+\Gamma_{0} C & A \Gamma_{1}+\Gamma_{0} D
\end{array}\right) .
$$

Before we enter the somewhat lengthy discussion of the eigenvalues of $J$ for arbitrary dimension $N$, we consider the case of two coupled 1D maps, i.e., $N=1$, where the Hopf bifurcation now follows immediately. In this case $A, B, C, D, \Gamma_{0}$, and $\Gamma_{1}$ are $c$ numbers, and hence commute. As a consequence of (3.23) one thus gets for $J$ the simple expression

$$
J=\left[\begin{array}{ll}
\gamma & 0 \\
0 & \gamma
\end{array}\right]+\epsilon\left[\begin{array}{cc}
q & p \Gamma_{1} \\
p \Gamma_{0} & q
\end{array}\right]+O\left(\epsilon^{2}\right),
$$

where the abbreviations $q \equiv A \Gamma_{1}+D \Gamma_{0}$ and $p \equiv B+C$ have been used. $\gamma \equiv \Gamma_{0} \Gamma_{1}=\Gamma_{1} \Gamma_{0}$ is the stability coefficient of the single-system orbit $z_{0}, z_{1}$. The eigenvalues of $J$ are given by

$$
\eta_{1,2}=\gamma+\epsilon(q \pm p \sqrt{\gamma})+O\left(\epsilon^{2}\right) .
$$

This is the same type of expression we already obtained for the coupled logistic maps in (2.20). Because the single system is assumed to undergo a period-doubling bifurcation at $\lambda=\lambda_{2}, \gamma$ is close to -1 for $\lambda$ close to $\lambda_{2}$ and thus negative. Therefore $\sqrt{\gamma}$ is purely imaginary and the eigenvalues $\eta_{1,2}$ are complex conjugate up to order $\epsilon$. The inclusion of higher orders in $\epsilon$ could change this result only if the sum of higher-order terms in $\epsilon$ could remove the imaginary part already present in order $\epsilon$ so that $\eta_{1,2}$ become real. (Different imaginary parts in $\eta_{1,2}$ are not allowed, because $J$ is a real matrix.) Even if this were the case for a given value of $\epsilon$, an infinitesimal change in $\epsilon$ would make the imaginary part of $\eta_{1,2}$ once more unequal to zero, because the higher-order terms have a different dependence on $\epsilon$ than the term of linear order in $\epsilon$. So generically complex conjugation of $\eta_{1,2}$ in order $\epsilon$ implies complex conjugation in all orders.

Moreover from (3.31) one obtains

$$
\left|\eta_{1,2}\right|=|\gamma|+\epsilon q+O\left(\epsilon^{2}\right) .
$$

$\gamma$ and $q$ are functions of the control parameter $\lambda$. Expanding these at $\lambda_{2}$ in the deviation $\Delta \lambda \equiv \lambda-\lambda_{2}$ one obtains

$$
\left|\eta_{1,2}\right|=1+\epsilon q_{2}+\gamma^{\prime} \Delta \lambda+O\left(\epsilon^{2}, \epsilon \Delta \lambda,(\Delta \lambda)^{2}\right),
$$

with $q=q_{2}+O(\Delta \lambda)$ and $|\lambda|=1+\gamma^{\prime} \Delta \lambda+O(\Delta \lambda)^{2}$. So for given $\epsilon<<1$ it is always possible to change $\Delta \lambda$ so that the modulus of $\eta_{1,2}$ crosses 1 . Remembering that $\eta_{1,2}$ are complex conjugate this means that $\eta_{1,2}$ cross the unit circle when $\lambda$ is varied appropriately. For the generic nonresonant case we have hereby shown that the considered antiphase orbit of coupled 1D maps undergoes a Hopf bi- 
furcation.

We now continue with the general case of arbitrary dimension $N$. We will once more arrive at an equation like (3.31) so that all conclusions apply.

The matrices $\Gamma_{1} \Gamma_{0}$ and $\Gamma_{0} \Gamma_{1}$ appearing in $U$ have the same eigenvalues, because both are a stability matrix of the period-2 orbit $z_{0}, z_{1}$ of $f_{\lambda}$ :

$$
\Gamma_{1} \Gamma_{0}=\left.\frac{\partial f_{\lambda}^{2}}{\partial x}\right|_{z_{0}} \text { and } \Gamma_{0} \Gamma_{1}=\left.\frac{\partial f_{\lambda}^{2}}{\partial x}\right|_{z_{1}} .
$$

We intend to use once more Proposition 1 from the Appendix for the computation of eigenvalues. To this end we introduce a coordinate transformation $K$ that diagonalizes $U$. In the generic situation $\Gamma_{1} \Gamma_{0}$ and $\Gamma_{0} \Gamma_{1}$ are diagonalizable. Let $K_{0}$ and $K_{1}$ denote $N \times N$ matrices that diagonalize $\Gamma_{1} \Gamma_{0}$ and $\Gamma_{0} \Gamma_{1}$, respectively. Because these stability matrices have the same eigenvalues one can write

$$
\Gamma \equiv\left(\begin{array}{ccc}
\gamma_{1} & & 0 \\
& \ddots & \\
0 & & \gamma_{N}
\end{array}\right)=K_{0}^{-1} \Gamma_{1} \Gamma_{0} K_{0}=K_{1}^{-1} \Gamma_{0} \Gamma_{1} K_{1} .
$$

Therefore the coordinate transformation

$$
K \equiv\left(\begin{array}{cc}
K_{0} & 0 \\
0 & K_{1}
\end{array}\right)
$$

diagonalizes $U$ and one obtains

$$
J^{\prime}=K^{-1} J K=U^{\prime}+\epsilon V^{\prime}+O\left(\epsilon^{2}\right)
$$

with

$$
U^{\prime}=\left(\begin{array}{ll}
\Gamma & 0 \\
0 & \Gamma
\end{array}\right) \text { and } V^{\prime}=\left(\begin{array}{cc}
\Gamma_{1}^{\prime} A^{\prime}+D^{\prime} \Gamma_{0}^{\prime} & \Gamma_{1}^{\prime} B^{\prime}+C^{\prime} \Gamma_{1}^{\prime} \\
B^{\prime} \Gamma_{0}^{\prime}+\Gamma_{0}^{\prime} C^{\prime} & A^{\prime} \Gamma_{1}^{\prime}+\Gamma_{0}^{\prime} D^{\prime}
\end{array}\right),
$$

where we introduced

$$
\begin{array}{lll}
A^{\prime} \equiv K_{1}^{-1} A K_{0}, & C^{\prime} \equiv K_{0}^{-1} C K_{0}, & \Gamma_{0}^{\prime} \equiv K_{1}^{-1} \Gamma_{0} K_{0}, \\
B^{\prime} \equiv K_{1}^{-1} B K_{1}, & D^{\prime} \equiv K_{0}^{-1} D K_{1}, & \Gamma_{1}^{\prime} \equiv K_{0}^{-1} \Gamma_{1} K_{1} .
\end{array}
$$

$U^{\prime}$ is diagonal because $\Gamma$ is diagonal. Therefore Proposition 1 from the Appendix can be applied. All eigenvalues $\gamma_{1}, \ldots, \gamma_{N}$ appear twice in $\Gamma$. Without loss of generality it can be assumed that the eigenvalue $\gamma_{1}$ produces the period doubling in the single map. This $\gamma_{1}$ appears in $U^{\prime}$ at the positions $\left(U^{\prime}\right)_{1,1}$ and $\left(U^{\prime}\right)_{N+1, N+1}$. Therefore one has from Proposition 1 that the related eigenvalues $\eta_{1,2}$ of $J$ up to order $\epsilon$ are given by

$$
\eta_{1,2}=\gamma_{1}+\epsilon \kappa_{1,2}+O\left(\epsilon^{2}\right),
$$

where $\kappa_{1,2}$ are eigenvalues of the submatrix

$$
\begin{aligned}
V_{\mathrm{sub}}^{\prime} & \equiv\left[\begin{array}{cc}
\left(V^{\prime}\right)_{1,1} & \left(V^{\prime}\right)_{1, N+1} \\
\left(V^{\prime}\right)_{N+1,1} & \left(V^{\prime}\right)_{N+1, N+1}
\end{array}\right] \\
& =\left[\begin{array}{ll}
\left(\Gamma_{1}^{\prime} A^{\prime}+D^{\prime} \Gamma_{0}^{\prime}\right)_{1,1} & \left(\Gamma_{1}^{\prime} B^{\prime}+C^{\prime} \Gamma_{1}^{\prime}\right)_{1,1} \\
\left(B^{\prime} \Gamma_{0}^{\prime}+\Gamma_{0}^{\prime} C^{\prime}\right)_{1,1} & \left(A^{\prime} \Gamma_{1}^{\prime}+\Gamma_{0}^{\prime} D^{\prime}\right)_{1,1}
\end{array}\right]
\end{aligned}
$$

of $V^{\prime}$. From the viewpoint of bifurcation theory this reduction is the reduction to the center manifold [34].

Our next aim is to determine the eigenvalues $\kappa_{1,2}$ of

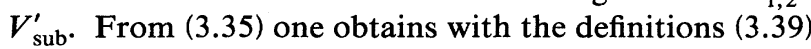

$$
\Gamma=\Gamma_{1}^{\prime} \Gamma_{0}^{\prime}=\Gamma_{0}^{\prime} \Gamma_{1}^{\prime} \text {. }
$$

so that in these new coordinates both stability matrices of the single-system period- 2 orbit are diagonal. This equation implies that $\Gamma_{0}^{\prime}$ and $\Gamma_{1}^{\prime}$ can be simultaneously diagonalized by a simple permutation of indices (see Proposition 2 in the Appendix). Without loss of generality it can be assumed that this permutation is contained in the coordinate transformations $K_{0}$ and $K_{1}$ so that $\Gamma_{0}^{\prime}$ and $\Gamma_{1}^{\prime}$ are already diagonal:

$$
\Gamma_{0}^{\prime}=\left(\begin{array}{ccc}
\alpha_{1} & & 0 \\
& \ddots & \\
0 & & \alpha_{N}
\end{array}\right) \Gamma_{1}^{\prime}=\left(\begin{array}{ccc}
\beta_{1} & & 0 \\
& \ddots & \\
0 & & \beta_{N}
\end{array}\right) .
$$

Therefore one gets

$$
\begin{aligned}
q \equiv\left(\Gamma_{1}^{\prime} A^{\prime}+D^{\prime} \Gamma_{0}^{\prime}\right)_{1,1} & =\beta_{1} A_{1,1}^{\prime}+D_{1,1}^{\prime} \alpha_{1} \\
& =\left(A^{\prime} \Gamma_{1}^{\prime}+\Gamma_{0}^{\prime} D^{\prime}\right)_{1,1}
\end{aligned}
$$

and the diagonal elements of $V_{\text {sub }}^{\prime}$ are identical. For the nondiagonal elements one obtains

$\left(\Gamma_{1}^{\prime} B^{\prime}+C^{\prime} \Gamma_{1}^{\prime}\right)_{1,1}=\beta_{1} B_{1,1}^{\prime}+C_{1,1}^{\prime} \beta_{1}=\left(B_{1,1}^{\prime}+C_{1,1}^{\prime}\right) \beta_{1}=p \beta_{1}$

and

$\left(B^{\prime} \Gamma_{0}^{\prime}+\Gamma_{0}^{\prime} C^{\prime}\right)_{1,1}=B_{1,1}^{\prime} \alpha_{1}+\alpha_{1} C_{1,1}^{\prime}=\left(B_{1,1}^{\prime}+C_{1,1}^{\prime}\right) \alpha_{1}=p \alpha_{1}$

with

$$
p \equiv B_{1,1}^{\prime}+C_{1,1}^{\prime} .
$$

Thus $V_{\text {sub }}^{\prime}$ can be written as

$$
V_{\text {sub }}^{\prime}=\left(\begin{array}{cc}
q & p \beta_{1} \\
p \alpha_{1} & q
\end{array}\right)
$$

This structure of $V_{\text {sub }}^{\prime}$ is once more a result of (3.23). Remembering that $\Gamma=\Gamma_{0}^{\prime} \Gamma_{1}^{\prime}$ one has $\gamma_{1}=\alpha_{1} \beta_{1}$ and thus the eigenvalues of $V_{\text {sub }}^{\prime}$ are

$$
\kappa_{1,2}=q \pm p \sqrt{\gamma_{1}} \text {. }
$$

Entering this into (3.40) one obtains for the two eigenvalues $\eta_{1,2}$ of the stability matrix $J$

$$
\eta_{1,2}=\gamma_{1}+\epsilon\left(q \pm p \sqrt{\gamma_{1}}\right)+O\left(\epsilon^{2}\right) .
$$

This equation is the same as we obtained in the case of two coupled 1D maps (3.31). There we concluded from this equation that the eigenvalues $\eta_{1,2}$ are complex conjugate for $\lambda$ close to $\lambda_{2}$ and leave or enter the complex unit circle when $\lambda$ is changed appropriately so that the antiphase orbit undergoes a Hopf bifurcation. This same reasoning applies here if one can show that $q$ and $p$ are 
real numbers, because this was implicitly assumed. That $p$ and $q$ are real is not immediately obvious, because the coordinate transformation $K$ we performed to diagonalize the $\epsilon$-independent part $U$ of $J$ may have introduced complex numbers. In the following it will be explicitly shown that $p$ and $q$ are real, so that indeed from Eq. (3.50) the Hopf bifurcation follows.

We first show that $q$ is real. $K_{0}$ diagonalizes the matrix $\Gamma_{1} \Gamma_{0}$. If its right eigenvectors belonging to the eigenvalues $\gamma_{1}, \ldots, \gamma_{N}$ are denoted by $a_{1}^{(r)}, \ldots, a_{N}^{(r)}$, respectively, then $K_{0}$ is given by

$$
K_{0}=\left(a_{1}^{(r)}, a_{2}^{(r)}, \ldots, a_{N}^{(r)}\right) .
$$

The eigenvalue $\gamma_{1}$ is real and therefore the eigenvector $a_{1}^{(r)}$ is also real. The same arguments apply to the inverse $K_{0}^{-1}$, so that $K_{0}^{-1}$ is given by

$$
K_{0}^{-1}=\left(a_{1}^{(l)}, a_{2}^{(l)}, \ldots, a_{N}^{(l)}\right)^{T},
$$

where $a_{1}^{(l)^{T}}, \ldots, a_{N}^{(l)^{T}}$ are the left eigenvectors of $\Gamma_{1} \Gamma_{0}$ belonging to the eigenvalues $\gamma_{1}, \ldots, \gamma_{N}$. Because $\gamma_{1}$ is real, $a_{1}^{(l)^{T}}$ is also real. Now from (3.44) and (3.39) one has

$$
\begin{aligned}
q=\left(\Gamma_{1}^{\prime} A^{\prime}+D^{\prime} \Gamma_{0}^{\prime}\right)_{1,1}= & {\left[K_{0}^{-1}\left(\Gamma_{1} A+D \Gamma_{0}\right) K_{0}\right]_{1,1} } \\
= & a_{1}^{(l)}\left(\Gamma_{1} A+D \Gamma_{0}\right) a_{1}^{(r)}
\end{aligned}
$$

This last equation shows that $q$ is real, because $\Gamma_{1} A+D \Gamma_{0}$ and $a_{1}^{(l)}{ }^{T}$ and $a_{1}^{(r)}$ are real.

This same type of reasoning can be used to show that $p$ is real. $K_{1}$ diagonalizes $\Gamma_{0} \Gamma_{1}$ [see Eq. (3.35)]. Therefore $K_{1}$ and $K_{1}^{-1}$ can be written as

$$
K_{1}=\left(b_{1}^{(r)}, b_{2}^{(r)}, \ldots, b_{N}^{(r)}\right),
$$

and

$$
K_{1}^{-1}=\left(b_{1}^{(l)}, b_{2}^{(l)}, \ldots, b_{N}^{(l)}\right)^{T},
$$

where the $b_{k}^{(r)}$ and $b_{k}^{(l)}$ are right and left eigenvectors of $\Gamma_{0} \Gamma_{1}$ belonging to the eigenvalues $\gamma_{k} \cdot b_{1}^{(r)}$ and $b_{1}^{(l)^{T}}$ are real because $\gamma_{1}$ is real. From (3.47) together with (3.39)

$$
\begin{aligned}
p=B_{1,1}^{\prime}+C_{1,1}^{\prime} & =\left(K_{1}^{-1} B K_{1}\right)_{1,1}+\left(K_{0}^{-1} C K_{0}\right)_{1,1} \\
& =b_{1}^{(l)^{T}} B b_{1}^{(r)}+a_{1}^{(l)} C a_{1}^{(r)} .
\end{aligned}
$$

All quantities in this last equation are real, and so $p$ is also real. This finishes the proof that generically the antiphase orbit undergoes a Hopf bifurcation.

\section{Extension to higher-order period doublings}

So far we considered the consequences of a symmetric coupling of systems with a fixed point that undergoes two subsequent period doublings. The first period doubling produces an in-phase and an antiphase orbit in the coupled system and the second period doubling is responsible for the Hopf bifurcation of the antiphase orbit. Similar phenomena exist for symmetrically coupled systems with two subsequent period-doubling bifurcations from a periodic orbit of arbitrary period.

For example, in the case of coupled logistic maps [see Figs. 1(a) and 1(b)] one sees in the coupled system at $\lambda \approx 3.45$ a symmetric period- 4 orbit that undergoes at $\lambda \approx 3.5$ a period doubling to an antiphase period -8 orbit that becomes unstable by a Hopf bifurcation at $\lambda \approx 3.52$. In the single system one sees instead a period- 2 orbit that undergoes two subsequent period-doubling bifurcations. Another example is shown in Figs. 6(a) and 6(b), where part of the period-3 window of the logistic map is plotted for the single system and the symmetrically coupled system. The first two period doublings of the period-3 orbit change in the coupled system to a period doubling followed by a Hopf bifurcation.

This behavior is a direct consequence of the considerations from the last section. It is easy to show that the $p$ times iterated system (3.1) is once more symmetric. So a symmetric period-p orbit of the original system results in $p$ symmetric fixed points of the iterated system. And if the period- $p$ orbit of the original system undergoes two subsequent period doublings, each symmetric fixed point of the iterated system will do the same. So all results from the previous section carry over to the bifurcations of symmetric orbits of arbitrary period. Note that the existence of a period- $p$ orbit in the single system not only implies the existence of a symmetric period-p orbit in the coupled system, but also the existence of nonsymmetric period- $p$ orbits. But our considerations are concerned only with the bifurcations emerging from a symmetric orbit.
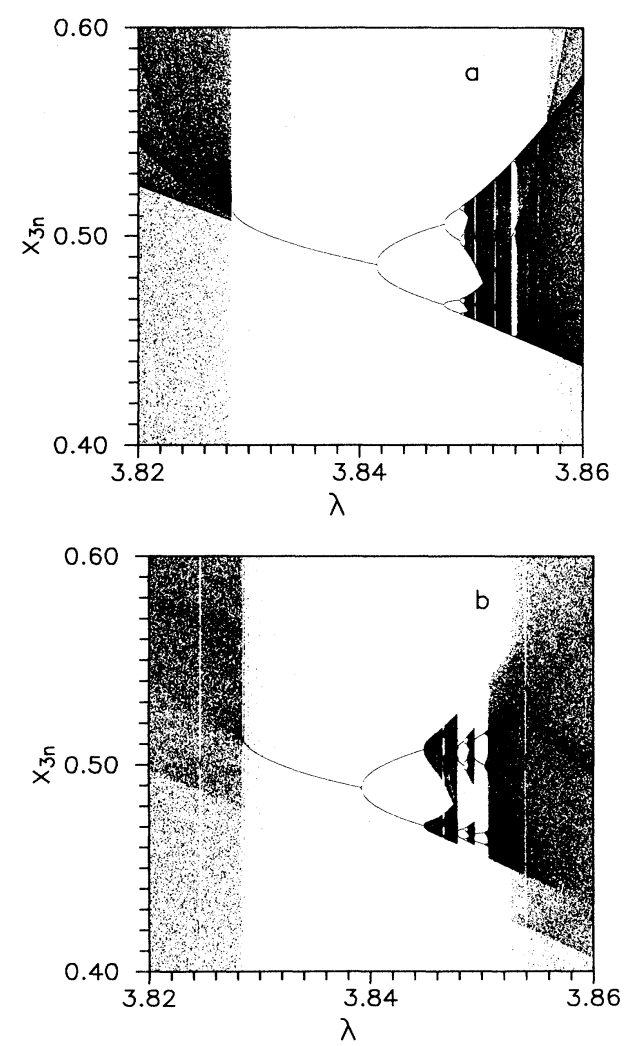

FIG. 6. Symmetrically coupled logistic maps [Eqs. (2.1) and (2.2)]: the period-3 window. (a) Uncoupled: $\epsilon=0$; (b) coupled: $\epsilon=-0.01$. 


\section{Small asymmetry}

Until now we have only considered coupled maps that are symmetric under the exchange of $x$ and $y$. But in macroscopic systems, to which our considerations apply, symmetries are never perfect. So if the bifurcations so far proved for symmetric systems are a real world phenomenon, they should be stable against small asymmetric perturbations. This is indeed the case, as will be shown in the following.

To this end the following extended system is considered:

$x_{n+1}=f_{\lambda}\left(x_{n}\right)+\epsilon g_{\epsilon}\left(x_{n}, y_{n}\right)+\kappa n_{\kappa, \epsilon}\left(x_{n}, y_{n}\right)$,

$y_{n+1}=f_{\lambda}\left(y_{n}\right)+\epsilon g_{\epsilon}\left(y_{n}, x_{n}\right)+\kappa m_{\kappa, \epsilon}\left(x_{n}, y_{n}\right)$

with the symmetry breaking parameter $\kappa,|\kappa| \ll<1$, and the symmetry breaking functions $n_{\kappa, \epsilon}$ and $m_{\kappa, \epsilon}$ that are assumed to be analytic in $\kappa$ and $\epsilon$ at $\kappa=\epsilon=0$.

First the bifurcations of the initial fixed point are considered. By the introduction of the small asymmetry the eigenvalues of the stability matrix $J$ of the fixed point are given by

$$
\widetilde{\eta}_{1,2}=\eta_{1,2}+\kappa \zeta_{1,2}+O\left(\epsilon^{2}, \epsilon \kappa, \kappa^{2}\right),
$$

where $\eta_{1,2}(\epsilon)$ are the eigenvalues (3.15) obtained in the symmetric case. Because we consider only real mappings, the eigenvalues $\widetilde{\eta}_{1,2}$ are both real or complex conjugate. In order to show that in the asymmetric case the bifurcations of the fixed point are still period-doubling bifurcations, one has to exclude the latter possibility. To this end we recall that for $\lambda$ close to $\lambda_{1}$ the eigenvalues $\eta_{1,2}$ of the symmetric case are real and-in the generic case-differ. So it is obvious that, whatever the values of $\zeta_{1,2}$ might be, the real parts of $\widetilde{\eta}_{1}$ and $\widetilde{\eta}_{2}$ can be identical only for very specific choices of $\kappa$. Therefore $\widetilde{\eta}_{1}$ and $\widetilde{\eta}_{2}$ typically cannot be complex conjugate and thus in the generic case are real valued. The same arguments hold when higher orders in $\epsilon$ and $\kappa$ are included. This shows that the bifurcations of the fixed point in the symmetric and weakly asymmetric cases are the same; only the values of $\lambda$ where the bifurcations appear are shifted by an amount depending on $\kappa$.

Next the Hopf bifurcation of the antiphase orbit is considered. In the strict sense of Eq. (2.15), an antiphase orbit no longer exists in the asymmetric system. Nevertheless - as we saw above - the bifurcation structure of the original fixed point has not changed, and there is a period- 2 orbit in the asymmetric system that has the antiphase property $(2.15)$ in the limit $\kappa \rightarrow 0$. So for $\kappa \neq 0$ this orbit obeys $(2.15)$ in an approximate sense and we continue to call this orbit the "antiphase" orbit.

The eigenvalues $\widetilde{\eta}_{1,2}$ of the antiphase orbit in the asymmetric system typically differ from the respective eigenvalues $\widetilde{\eta}_{1,2}$ in the symmetric system in order $\kappa$ :

$$
\widetilde{\eta}_{1,2}=\eta_{1,2}+\kappa \xi_{1,2}+O\left(\epsilon^{2}, \epsilon \kappa, \kappa^{2}\right) \text {. }
$$

This is formally the same relation as (3.57), but here, for $\lambda$ close to the Hopf bifurcation in the symmetric system, the eigenvalues $\widetilde{\eta}_{1,2}$ are complex conjugate. Now a similar reasoning as above reveals that the corrections $\zeta_{1,2}$ in- troduced by the asymmetry can remove the imaginary parts of $\eta_{1,2}$ only for very specific choices of $\kappa$. Therefore in the generic case the eigenvalues $\widetilde{\eta}_{1,2}$ are complex conjugate-even if higher orders in $\kappa$ are considered - so that the Hopf bifurcation of the antiphase orbit persists.

These considerations show that even for small asymmetry one of the bifurcation diagrams Figs. 5(b) or 5(c) applies.

\section{TWO IDENTICAL, SYMMETRICALLY COUPLED SYSTEMS OF DIFFERENTIAL EQUATIONS}

We saw in Fig. 3 that two symmetrically coupled Rössler systems behave as symmetrically coupled maps. In this section we show that this is not incidental, but generally true. There is only one minor difference compared to coupled maps: In differential equations a periodic solution emerges from a fixed point not by a period-doubling-bifurcation but by a Hopf bifurcation. So in addition to the case of two subsequent perioddoubling bifurcations in the single system, one has to consider here the case of a Hopf bifurcation followed by a period-doubling bifurcation.

More explicitly we consider the symmetrically coupled system

$$
\begin{aligned}
& \dot{x}=F_{\lambda}(x)+\epsilon G_{\epsilon}(x, y), \\
& \dot{y}=F_{\lambda}(y)+\epsilon G_{\epsilon}(y, x)
\end{aligned}
$$

with $x, y \in \mathbb{R}^{N}$ and $\epsilon$ small. It is assumed that the single system $\dot{x}=F_{\lambda}(x)$ undergoes two subsequent bifurcations at $\lambda=\lambda_{1}$ and $\lambda=\lambda_{2}$. The first of these bifurcations can be either a Hopf bifurcation (if a fixed point gets unstable) or a period-doubling bifurcation (if a periodic orbit gets unstable). The second bifurcation is always a perioddoubling bifurcation [compare Fig. 5(a)]. Under these conditions we show that generically-depending on the sign of the coupling parameter $\epsilon$ - one of the bifurcation diagrams $5(\mathrm{~b})$ or $5(\mathrm{c})$ holds. More specifically it will be proved that the first bifurcation in the single system gives rise to two bifurcations of the same type in the coupled system, one resulting in a periodic solution that is symmetric, the other resulting in a nonsymmetric solution with the generalized antiphase property

$$
\mathcal{S}(x(t), y(t))=\left[x\left[t+\frac{T}{2}\right), y\left[t+\frac{T}{2}\right]\right),
$$

where $T$ is the period of the solution and $\mathcal{S}$ the symmetry operation $x \leftrightarrow y$. Concerning the second bifurcation we show that generically the antiphase solution undergoes a Hopf bifurcation.

The case where the first single-system bifurcation is a Hopf bifurcation is much simpler than the other possibility of a period-doubling bifurcation. Therefore we consider this case first. In subsection IV A we show that the Hopf bifurcation of the single system gives rise to two Hopf bifurcations in the coupled system, one producing an in-phase solution the other an antiphase solution. We continue in subsection IV $B$ by showing that the second bifurcation in the single system-the period-doubling bifurcation - changes into a Hopf bifurcation of the anti- 
phase solution of the coupled system. Here we partially take over results from Sec. III. Thereby the proof for the first case of bifurcations is complete.

In subsection IV $C$ we then consider the remaining case, where the first single-system bifurcation is a perioddoubling bifurcation. It will be shown that the singlesystem period-doubling bifurcation gives rise to two period-doubling bifurcations in the coupled system, where once more one solution is symmetric, whereas the other has the antiphase property. This completes our proof of the validity of the bifurcation diagrams $5(\mathrm{~b})$ or 5(c). Nevertheless, we finally consider in subsection IV D briefly the influence of a small nonsymmetry, in order to make sure that our results are relevant to macroscopic systems.

\section{A. The first bifurcation is a Hopf bifurcation}

For $\lambda<\lambda_{1}$ the single system has a fixed point $z$. Therefore the uncoupled system has a symmetric fixed point $(z, z)$. For $\epsilon \neq 0,|\epsilon|$ sufficiently small, this fixed point generically persists and remains symmetric because the coupling does not destroy the symmetry. Although the uncoupled system (and thus also the coupled system) may also have nonsymmetric fixed points, we are exclusively concerned with the bifurcations of the symmetric fixed point. Other fixed points may show sequences of bifurcations that are different from those of the symmetric fixed point.

In a Hopf bifurcation a pair of eigenvalues of the stability matrix crosses the imaginary axis. So the singlesystem Hopf bifurcation implies a degenerate Hopf bifurcation in the uncoupled system $(\epsilon=0)$, where two identical pairs of eigenvalues cross the imaginary axis. Because the system (4.1) is real the eigenvalues can only be real or come in complex-conjugate pairs. For finite imaginary part - the generic case - the multipliers cannot become real by introducing an infinitesimal coupling $\epsilon \neq 0$. Therefore with (sufficiently small) coupling there still exist two pairs of complex-conjugate multipliers. Generically their dependence on $\epsilon$ differs, and the degenerate Hopf bifurcation in the uncoupled system splits into two Hopf bifurcations in the coupled system.

In each of these two Hopf bifurcations a single periodic solution is created. If we denote such a periodic solution by $(x(t), y(t))$, then the symmetry of the system implies that $\mathcal{S}(x(t), y(t))$ is also a solution. But because only one single new solution is created, the symmetry operation $\mathcal{S}$ has to map this new solution $(x(t), y(t))$ onto itself, possibly with a phase shift $\tau$. So one has

$$
\mathcal{S}(x(t), y(t))=(y(t), x(t))=(x(t+\tau), y(t+\tau)) .
$$

If $(x(t), y(t))$ is symmetric, then $\tau=T$ (or equivalently an integer multiple of $T$ ), where $T$ is the period of the solution. When the solution is nonsymmetric, then, because twice the symmetry operation is the identity,

$\mathcal{S}^{2}(x(t), y(t))=(x(t), y(t))=(x(t+2 \tau), y(t+2 \tau))$.

So $2 \tau$ has to be an integer multiple of the period $T$ and one can take without loss of generality $\tau=T / 2$. Consequently, the nonsymmetric orbit emerging from the Hopf bifurcation has the antiphase property (4.2).

Next we show that one of the two Hopf bifurcations produces a symmetric solution and the other an antiphase solution. A periodic solution created in a Hopf bifurcation emerges in the eigenspace of the Jacobian $J$ that belongs to the eigenvalues that become unstable. Therefore the symmetry of the periodic orbit is intimately related to the way the symmetry operation acts on that eigenspace. This is well known from the theory of bifurcations in the presence of symmetries [21,35]. For the symmetry $\rho$ we are considering here, this relation can be obtained as follows. Consider first the case $\epsilon \neq 0$, where we have two nondegenerate Hopf bifurcations. If the periodic solution created in such a Hopf bifurcation is symmetric, then $\mathcal{S}(x(t), y(t))=(x(t), y(t))$, and we see that $\mathcal{S}$ does not change points of the solution. It acts as the identity on the related eigenspace: $\mathcal{S} r=r$ for vectors $r$ from the eigenspace. If, instead, the solution created in that eigenspace has the antiphase property

$$
\mathcal{S}(x(t), y(t))=(x(t+T / 2), y(t+T / 2)),
$$

then points from the related eigenspace are changed under the action of the symmetry operation so that $\mathcal{S} r \neq r$. But from the symmetry of the Jacobian $\mathcal{S} \mathscr{S}=J$ and the fact that the considered eigenspace is spanned by two complex-conjugate eigenvectors, one can conclude that the whole eigenspace has a definite symmetry: either $\mathcal{\rho} r=r$ or $\rho r=-r$. So with our previous result $\mathcal{S} \neq \neq r$ only the second relation is possible. These considerations so far show that an in-phase solution is related to an eigenspace of $J$ that is pointwise invariant under $\mathcal{S}$, whereas an antiphase solution is related to an eigenspace of $J$ that is flipped by $\mathcal{S}$. Next the case $\epsilon=0$ is considered. Here the system is uncoupled and one can show by explicit construction that the eigenspace of $J$ that goes unstable in the doubly degenerate Hopf bifurcation is spanned by a pair of eigenvectors of $\mathcal{S}$ belonging to the eigenvalue +1 (pointwise invariant) and a pair of eigenvectors belonging to the eigenvalue -1 (flip). Finally, by comparing this result with our previous result for $\epsilon \neq 0$ in the limit $\epsilon \rightarrow 0$, we conclude that they fit only if one of the two Hopf bifurcations produces an in-phase orbit and the other produces an antiphase orbit.

\section{B. Hopf bifurcation of the antiphase solution}

In this section it will be shown that, as a result of the period-doubling bifurcation of the single system, the antiphase solution undergoes a Hopf bifurcation. To this end we temporarily introduce a condensed notation that simplifies some of the calculations. Let $q \equiv(x, y)$. Then the coupled system (4.1) can be rewritten as

$$
\dot{q}=\mathscr{F}_{\lambda, \epsilon}(q),
$$

where $\mathscr{F}_{\lambda, \epsilon}$ is invariant under the symmetry operation $\mathcal{S}$ :

$$
\mathcal{S} \mathcal{F}_{\lambda, \epsilon} \mathcal{S}(q)=\mathscr{F}_{\lambda, \epsilon}(q) .
$$

Most objects introduced in the following depend on $\lambda$ and 
$\epsilon$. For simplicity we usually do not indicate this dependence and also omit the indices on $\mathcal{F}$.

The flow of the system (4.5) will be denoted by $\Phi_{t}(q)$. For a point $q_{t}$ from the antiphase solution one has according to (4.2)

$$
\Phi_{T / 2}\left(q_{t}\right)=\mathfrak{S} q_{t} \text {. }
$$

The stability of this antiphase solution is determined by the eigenvalues of the stability matrix

$$
J=\left.\frac{\partial \Phi_{T}(q)}{\partial q}\right|_{q=q_{0}},
$$

where $q_{0}$ is here and in the following a given point of the antiphase solution. In order to take advantage of the antiphase property we define

$$
\left.J_{0} \equiv \frac{\partial \Phi_{T / 2}(q)}{\partial q}\right|_{q_{0}} \text { and }\left.J_{1} \equiv \frac{\partial \Phi_{T / 2}(q)}{\partial q}\right|_{q_{1}}
$$

with $q_{1} \equiv \Phi_{T / 2}\left(q_{0}\right)=S q_{0}$ another point on the antiphase orbit. With $\Phi_{T}(q)=\Phi_{T / 2}\left(\Phi_{T / 2}(q)\right)$ one finds

$$
J=J_{1} J_{0} \text {. }
$$

Next we prove as a counterpart of (3.22) the relation

$$
J_{1}=\rho J_{0} \rho \text {. }
$$

If the derivative of the flow is denoted by

$$
\left.J_{t}(q) \equiv \frac{\partial \Phi_{t}(q)}{\partial q}\right|_{q},
$$

then one obtains from (4.5)

$$
\frac{d}{d t} J_{t}\left(q_{0}\right)=\left.\frac{\partial \mathscr{F}(q)}{\partial q}\right|_{\Phi_{t}\left(q_{0}\right)} J_{t}\left(q_{0}\right)
$$

and the analogous equation

$$
\frac{d}{d t} J_{t}\left(q_{1}\right)=\left.\frac{\partial \mathcal{F}(q)}{\partial q}\right|_{\Phi_{t}\left(q_{1}\right)} J_{t}\left(q_{1}\right) .
$$

With the initial conditions

$$
J_{t=0}\left(q_{0}\right)=J_{t=0}\left(q_{1}\right)=\mathbb{1},
$$

that are a consequence of the definition (4.12) of $J_{t}$, these equations uniquely determine $J_{t}\left(q_{i}\right)$ because the original differential equation (4.5) as a model for a physical system uniquely determines the flow. Now the symmetry of $\mathcal{F}$ implies

$$
\begin{aligned}
& \left.\frac{\partial \mathcal{F}(q)}{\partial q}\right|_{\Phi_{t}\left(q_{1}\right)}=\left.\frac{\partial}{\partial q}[\mathcal{S F} \mathcal{S}(q)]\right|_{\Phi_{t}\left(q_{1}\right)} \\
& =\left.\mathcal{S} \frac{\partial \mathcal{F}(q)}{\partial q}\right|_{\mathcal{S} \Phi_{t}\left(q_{1}\right)} \mathcal{\rho} \\
& =\left.\mathcal{S} \frac{\partial \mathcal{F}(q)}{\partial q}\right|_{\Phi_{t}\left(q_{0}\right)} \mathcal{S} .
\end{aligned}
$$

Therefore operating $\delta$ from left and right on (4.13) gives

$$
\frac{d}{d t}\left[\mathcal{S} J_{t}\left(q_{0}\right) \mathcal{S}\right]=\left.\frac{\partial \mathscr{F}(q)}{\partial q}\right|_{\Phi_{t}\left(q_{1}\right)}\left[\mathcal{S} J_{t}\left(q_{0}\right) \mathcal{S}\right] \text {. }
$$

Because for $t=0$ one has $\mathcal{S} J_{t=0}\left(q_{0}\right) \mathcal{S}=\mathbb{1}$ a comparison of this equation with $(4.14)$ reveals by the uniqueness of its solutions

$$
J_{t}\left(q_{1}\right)=\rho J_{t}\left(q_{0}\right) \rho .
$$

Therefore the derivative of the flow along the antiphase solutions has also a kind of antiphase property. With the obvious identities $J_{T / 2}\left(q_{0}\right)=J_{0}$ and $J_{T / 2}\left(q_{1}\right)=J_{1}$ the specialization of (4.18) to $t=T / 2$ finally proves (4.11).

Coming back to our original notation, where the variables $x$ and $y$ of the subsystems show up separately, one can formally write for the flow of the coupled system

$$
\Phi_{t}(x, y)=\left[\begin{array}{l}
\Theta_{t}(x)+\epsilon \varphi_{t}^{(\epsilon)}(x, y) \\
\Theta_{t}(y)+\epsilon \psi_{t}^{(\epsilon)}(x, y)
\end{array}\right) .
$$

Here $\Theta_{t}$ denotes the single-system flow, so that (4.19) has the correct limit for $\epsilon \rightarrow 0$. The new functions $\varphi_{t}^{(\epsilon)}$ and $\psi_{t}^{(\epsilon)}$ are implicitly defined by (4.19). Once more the dependence of all these functions on the control parameter $\lambda$ is not explicitly indicated.

For the point $q_{0}$ of the antiphase solution we write $q_{0}=\left(\bar{z}_{0}, \bar{z}_{1}\right)$. Then for the Jacobian $J_{0}$ defined in (4.9) one obtains

$$
\begin{aligned}
J_{0}= & {\left[\begin{array}{cc}
\left.\frac{\partial \Theta_{T / 2}}{\partial x}\right|_{\bar{z}_{0}} & 0 \\
0 & \left.\frac{\partial \Theta_{T / 2}}{\partial y}\right|_{\bar{z}_{1}}
\end{array} \mid\right.} \\
& +\epsilon\left|\begin{array}{cc}
\frac{\partial \varphi_{t}^{(\epsilon)}}{\partial x} \\
\frac{\partial \psi_{t}^{(\epsilon)}, \bar{z}_{1}}{\partial x} & \left.\frac{\partial \varphi_{t}^{(\epsilon)}}{\partial y}\right|_{\bar{z}_{0}, \bar{z}_{1}}, \frac{\partial \bar{z}_{t}}{\partial y}
\end{array}\right|
\end{aligned}
$$

Expanding the point $\left(\bar{z}_{0}, \bar{z}_{1}\right)$ of the antiphase orbit of the coupled system in $\epsilon$ around a nearby point $\left(z_{0}, z_{1}\right)$ of the antiphase orbit of the uncoupled system, the matrix $J_{0}$ reads

$$
J_{0}=\left[\begin{array}{cc}
\Gamma_{0} & 0 \\
0 & \Gamma_{1}
\end{array}\right]+\epsilon\left(\begin{array}{ll}
A & B \\
C & D
\end{array}\right)+O\left(\epsilon^{2}\right),
$$

where

$$
\left.\Gamma_{0} \equiv \frac{\partial \Theta_{T / 2}}{\partial x}\right|_{z_{0}} \text { and }\left.\Gamma_{1} \equiv \frac{\partial \Theta_{T / 2}}{\partial x}\right|_{z_{1}} .
$$

The details of the matrices $A, B, C$, and $D$ are not needed in the following. The meaning of $\Gamma_{0}$ and $\Gamma_{1}$ becomes obvious when recognizing that the eigenvalues of the matrix

$$
\left.\frac{\partial \Theta_{T}(x)}{\partial x}\right|_{z_{0}}=\left.\frac{\partial \Theta_{T / 2}\left(\Theta_{T / 2}(x)\right)}{\partial x}\right|_{z_{0}}=\Gamma_{1} \Gamma_{0}
$$


determine the stability of the single-system solution $z(t)=\Theta_{t}\left(z_{0}\right)$.

With (4.10) and (4.11) the stability of the antiphase solution of the coupled system up to order $\epsilon$ is completely determined by the expression (4.21) for $J_{0}$ :

$$
J=J_{1} J_{0}=\rho J_{0} \rho J_{0}=U+\epsilon V+O\left(\epsilon^{2}\right)
$$

with

$$
U=\left(\begin{array}{cc}
\Gamma_{1} \Gamma_{0} & 0 \\
0 & \Gamma_{0} \Gamma_{1}
\end{array}\right)
$$

and

$$
V=\left(\begin{array}{cc}
\Gamma_{1} A+D \Gamma_{0} & \Gamma_{1} B+C \Gamma_{1} \\
B \Gamma_{0}+\Gamma_{0} C & A \Gamma_{1}+\Gamma_{0} D
\end{array}\right) .
$$

This stability matrix has the same structure as that we obtained in Sec. III in the context of coupled maps [compare Eqs. (3.28) and (3.29)]. There we showed that because both single-system stability matrices $\Gamma_{1} \Gamma_{0}$ and $\Gamma_{0} \Gamma_{1}$ have an eigenvalue that passes -1 at $\lambda=\lambda_{2}$, the matrix $J$ generically has a pair of complex-conjugate eigenvalues passing the complex unit circle when $\lambda$ is changed appropriately. Because here once more $\Gamma_{1} \Gamma_{0}$ and $\Gamma_{0} \Gamma_{1}$ are the single-system stability matrices with an eigenvalue passing -1 at $\lambda_{2}$, the same conclusion can be drawn here. With this result we have shown that the antiphase orbit of the coupled system (4.1) generically undergoes a Hopf bifurcation.

With the result of Sec. IV A this completes the proof that one of the bifurcation diagrams Fig. 5(b) or 5(c) holds if the first bifurcation in the single system is a Hopf bifurcation.

\section{The first bifurcation is a period-doubling bifurcation}

In this section we show that, if the first bifurcation in the single system is a period-doubling bifurcation instead of a Hopf bifurcation, once more one of the bifurcation diagrams $5(\mathrm{~b})$ or $5(\mathrm{c})$ applies to the coupled system. To this end it is sufficient to show that the single-system period-doubling bifurcation gives rise to two perioddoubling bifurcations in the coupled system, one leading to an in-phase solution and the other to an antiphase solution. This is sufficient because it is already known from the previous section that the antiphase orbit, as the result of the second single-system (period-doubling) bifurcation, undergoes a Hopf bifurcation.

A periodic orbit $x(t)$ in the single system implies an infinity of periodic orbits $(x(t), x(t+\tau)), \tau$ arbitrary, in the uncoupled system. If the coupling $\epsilon$ is made nonzero, the translational invariance in $t$ in the two subsystems is no longer independent. Therefore the coupling dramatically reduces the number of possible solutions [17]. Because of the symmetry of the system only two types of possible solutions remain: the in-phase and antiphase solutions. We are concerned in the following exclusively with the sequence of bifurcations arising from an inphase solution.

For such an in-phase solution $q_{t}=(x(t), y(t))$ one has in analogy to Eq. (4.7) the following symmetry of the flow:

$$
\Phi_{T}\left(q_{t}\right)=\rho q_{t}
$$

where $T$ is the period of $q_{t}$. At a given point $q_{0}$ of the solution $q_{t}$ the stability matrix reads

$$
\left.J \equiv \frac{\partial \Phi_{T}}{\partial q}\right|_{q_{0}}
$$

Because of (4.26) $J$ obeys

$$
J=\rho J \rho \text {. }
$$

Once more this is Eq. (3.6) obtained in the context of maps.

Now $J$ depends on $\epsilon$ and can be expanded in the uncoupled case $\epsilon=0$ :

$$
J=U+\epsilon V+O\left(\epsilon^{2}\right),
$$

where

$$
U=\left(\begin{array}{ll}
\Gamma & 0 \\
0 & \Gamma
\end{array}\right)
$$

and $\Gamma$ is the stability matrix of the single-system periodic orbit. Because the symmetry (4.26) is independent of $\epsilon$, each term in (4.29) has to obey the same symmetry. Therefore a short calculation using the representation (3.7) of $\mathcal{S}$ shows that $V$ can be written as

$$
V=\left(\begin{array}{ll}
A & B \\
B & A
\end{array}\right) \text {. }
$$

Hence up to order $\epsilon$ one has

$$
J=\left(\begin{array}{ll}
\Gamma & 0 \\
0 & \Gamma
\end{array}\right)+\epsilon\left[\begin{array}{ll}
A & B \\
B & A
\end{array}\right]+O\left(\epsilon^{2}\right) .
$$

This is the same expression as (3.8) and (3.9) obtained in Sec. IV $A$ in the case of coupled maps. And because here also the single-system period-doubling bifurcation implies a real eigenvalue of $\Gamma$ that passes -1 at $\lambda=\lambda_{1}$, the same conclusions for the eigenvalues of $J$ apply; namely, for sufficiently small $\epsilon$, the eigenvalues of $J$ remain real and generically differ. So the degenerate period-doubling bifurcation in the single system splits into two perioddoubling bifurcations in the coupled system.

Following the same line of reasoning as in Sec. IV A, one can finally show that one of these period doublings leads to an in-phase solution, whereas the other leads to an antiphase solution. The period- $2 T$ solution is created in that manifold of the period- $T$ solution that becomes unstable. For $\epsilon \neq 0$ the related eigenspace of $J$ is generically one dimensional and thus spanned by a single eigenvector of $J$ and because of (4.28) this eigenvector of $J$ is also an eigenvector of $\mathcal{S}$ belonging to one of its eigenvalues +1 or -1 . If the period- $2 T$ solution is created in the " +1 " eigenspace, the solution is mapped point by point onto itself and is symmetric. If instead the solution is created in the " -1 " eigenspace, points of the period- $2 T$ solution are not mapped onto themselves and it is consequently (because of lack of other possibilities) an anti- 
phase solution. So once more in-phase and antiphase solutions are related to the way the symmetry operation acts on the eigenspace of $J$ that becomes unstable. For $\epsilon=0$ the period-doubling bifurcation is doubly degenerate and one can show by explicit construction that the eigenspace of $J$ becoming unstable in the bifurcation is spanned by an eigenvector belonging to the eigenvalue +1 of $\rho$ and an eigenvector belonging to the eigenvalue -1 of $\mathcal{S}$. So by continuity in $\epsilon$ one can conclude that for $\epsilon \neq 0$ the two simple period-doubling bifurcations must produce an in-phase and an antiphase orbit. Together with the result from the previous section that the antiphase solution undergoes a Hopf bifurcation, this completes the proof that also for the case of two subsequent period-doubling bifurcations in the single system one of the bifurcation diagrams Fig. 5(b) or Fig. 5(c) correctly describes the sequence of bifurcations in the weakly coupled system.

\section{Small asymmetry}

We now consider briefly the influence of a small asymmetry on the bifurcation structure so far proved for the symmetrically coupled system. As in the case of maps (Sec. III D) generically a sufficiently small asymmetry will not destroy this bifurcation structure.

To be more definite we consider here the extended system

$$
\begin{aligned}
& \dot{x}=F_{\lambda}(x)+\epsilon G_{\epsilon}(x, y)+\kappa N_{\kappa, \epsilon}(x, y), \\
& \dot{y}=F_{\lambda}(x)+\epsilon G_{\epsilon}(y, x)+\kappa M_{\kappa, \epsilon}(x, y),
\end{aligned}
$$

where $\kappa$ is the symmetry breaking parameter and the symmetry breaking functions $N_{\kappa, \epsilon}$ and $M_{\kappa, \epsilon}$ may depend on $\epsilon$ and $\kappa$. In order to show that the bifurcation structure is not changed for sufficiently small $\kappa$, one has to prove the persistence of all bifurcations shown in Fig. 5(b) and 5(c). For every bifurcation the eigenvalues of the related stability matrix can for sufficiently small $\kappa$ be written as

$$
\widetilde{\eta}_{1,2}=\eta_{1,2}+\kappa \xi_{1,2}+O\left(\epsilon^{2}, \kappa \epsilon, \kappa^{2}\right),
$$

where the $\eta_{1,2}$ are the eigenvalues of the stability matrix in the symmetric case. The same reasoning presented in Sec. III D for the asymmetric coupling of maps applies here. In short, the considered dynamical system is real. Therefore the eigenvalues $\widetilde{\eta}_{1,2}$ belong to one of two classes: either $\eta_{1}$ and $\eta_{2}$ are real or $\eta_{1}$ is the complex conjugate of $\eta_{2}$. The same holds for $\eta_{1,2}$. Now because generically $\eta_{1} \neq \eta_{2}$ it is easy to show that if $\eta_{1,2}$ belong to one class, $\widetilde{\eta}_{1,2}$ cannot belong to the other class. Therefore, $\eta_{1,2}$ and $\widetilde{\eta}_{1,2}$ belong to the same class. This means that for sufficiently small asymmetry $\kappa$, the type of bifurcation does not change.

\section{DISCUSSION}

In this paper we proved that in symmetrically coupled identical oscillators quasiperiodicity emerges, if the single oscillator exhibits two subsequent period-doubling bifurcations - in the case of systems of differential equa- tions the first bifurcation may be a Hopf bifurcation. This result depends on two restrictions. First it holds only for almost all systems and almost all symmetric couplings. For example, it may happen that in certain systems the antiphase orbit of the coupled system undergoes period-doubling bifurcation instead of a Hopf bifurcation. This irregular behavior cannot be excluded, but is, as our proofs show, nongeneric. The second restriction is related to the magnitude of the coupling parameter $\epsilon$ : our result holds only for sufficiently small $\epsilon$. On first sight this restriction looks rather severe, but actually it is not. Throughout the paper the scale for $\epsilon$ was not specifiedand it could not be specified because $\epsilon$ appears in a product with the coupling functions $(g, G)$, so that the magnitude of $\epsilon$ depends on the choice of the magnitudes for the coupling functions. What really counts for our proofs is not the magnitude of $\epsilon$, but whether the expansions in $\epsilon$ performed in the proofs converge. Therefore "sufficiently small" means that $\epsilon$ should be inside the radius of convergence for the expansions. This radius of convergence is determined by a critical value of $\epsilon$, where the coupling gets so strong that new bifurcations occur that destroy the solutions we discussed so far. Without such bifurcations the radius of convergence may extend to infinity.

We also showed that our results remain valid in the presence of sufficiently small symmetry breaking terms. This is important, because in macroscopic systems, to which our considerations apply, symmetries are never perfect. Concerning the admissible magnitude of the nonsymmetry $\kappa$, the same remarks as those for the coupling parameter $\epsilon$ apply.

Finally we apply our results to the coupling of two systems with a Feigenbaum transition to chaos. In this case there is a good chance that the Feigenbaum transition is replaced by a quasiperiodic transition. Whether this last transition occurs or not crucially depends on the order in which the in-phase and antiphase orbits appear in the coupled system as a function of the control parameter. If the antiphase orbit appears first, it is stable and the system undergoes a Hopf bifurcation typically followed by quasiperiodic motion and a quasiperiodic transition is possible. If instead the in-phase orbit appears first, this is stable and the decision is delayed to its next period doubling. Here the question is once more whether an inphase or antiphase orbit appears first, so that the system has once more the chance to change to a quasiperiodic transition instead of a period-doubling transition. Because the order by which in-phase and antiphase orbits appear depends on the sign of the coupling $\epsilon$ one could think that in this way the system had at any stage of the Feigenbaum cascade the same chance $\frac{1}{2}$ to change the type of transition so that the probability for this change would be $\Sigma_{1}^{\infty}\left(\frac{1}{2}\right)^{n}=1$ and thus almost sure. But this is wrong: due to the self-similarity of the Feigenbaum transition at the transition point, asymptotically the order of the appearance of in-phase and antiphase orbits is identical at all stages of the transition [36]. Therefore, if the change to quasiperiodic behavior did not appear at an early stage of the Feigenbaum cascade, this change becomes improbable. Nevertheless, these considerations show that the probability for a replacement of the 
Feigenbaum transition by a quasiperiodic transition is larger than $\frac{1}{2}$, so that there is a tendency for this replacement.

Independent of the question of the replacement of the Feigenbaum transition by a quasiperiodic transition our results show that the Feigenbaum cascade in the symmetrically coupled system (which may be unstable) is generically accompanied by an infinity of Hopf bifurcations and so these should appear in a renormalization description [36]. This is a qualitatively different feature of the period-doubling transition in higher-dimensional systems compared to the transition in 1D maps [1]. Whether this also holds for period-doubling transitions in strongly coupled or strongly asymmetric systems is not known.

\section{ACKNOWLEDGMENTS}

C.R. gratefully acknowledges the hospitality of the Dynamical Systems Group at the Center of Chaos and Turbulence Studies of the Technical University of Denmark.

\section{APPENDIX}

In the paper we made use of two propositions. These are proved in this Appendix.

Proposition 1. Let $D$ be a diagonal $N \times N$ matrix

$$
D=\left(\begin{array}{ccc}
\delta_{1} & & 0 \\
& \ddots & \\
0 & & \delta_{N}
\end{array}\right)
$$

and $A$ and $B$ two other $N \times N$ matrices related to $D$ by

$$
A=D+\epsilon B+O\left(\epsilon^{2}\right)
$$

where $\epsilon$ is a small parameter, $|\epsilon| \ll 1$. If $A$ is diagonalizable then the eigenvalues $\alpha_{k}$ of $A$ up to order $\epsilon$ can be computed as follows.

(1) If $\delta_{k}$ is a nondegenerate eigenvalue of $D$ then

$$
\alpha_{k}=\delta_{k}+\epsilon B_{k k}+O\left(\epsilon^{2}\right) \text {. }
$$

(2) If an eigenvalue $\delta$ of $D$ is $n$-fold degenerate, so that one has a sequence of indices $k_{1}<k_{2}<\cdots<k_{n}$ with $\delta_{k_{1}}=\delta_{k_{2}}=\cdots=\delta_{k_{n}} \equiv \delta$ then one has for the related eigenvalues of $A$

$$
\alpha_{k_{j}}=\delta+\epsilon \kappa_{j}+O\left(\epsilon^{2}\right), j=1, \ldots, n,
$$

where the $\kappa_{j}$ are eigenvalues of the following submatrix of $B$ :

$$
\boldsymbol{B}_{\mathrm{sub}}=\left(\begin{array}{cccc}
\boldsymbol{B}_{k_{1} k_{1}} & \boldsymbol{B}_{k_{1} k_{2}} & \cdots & \boldsymbol{B}_{k_{1} k_{N}} \\
\vdots & & & \vdots \\
\boldsymbol{B}_{k_{N} k_{1}} & \cdots & \cdots & \boldsymbol{B}_{k_{N} k_{N}}
\end{array}\right) .
$$

Proof. Let $K$ be a coordinate transformation that diagonalizes $A$ up to order $\epsilon$ :

$$
K^{-1} A K=D+\epsilon D_{1}+O\left(\epsilon^{2}\right)
$$

with

$$
D_{1}=\left(\begin{array}{ccc}
\kappa_{1} & & 0 \\
& \ddots & \\
0 & & \kappa_{N}
\end{array}\right)
$$

diagonal. So $A$ has the eigenvalues $\alpha_{k}=\delta_{k}+\epsilon \kappa_{k}$ $+O\left(\epsilon^{2}\right)$. For $K$ we make the ansatz

$$
K=M(\mathbb{1}+\epsilon L)+O\left(\epsilon^{2}\right) .
$$

Entering this into (A6) gives with $K^{-1}=(\mathbb{1}-\epsilon L) M^{-1}$ $+O\left(\epsilon^{2}\right)$ the two equations

$$
\begin{aligned}
& M^{-1} D M=D, \\
& D L-L D+M^{-1} B M=D_{1} .
\end{aligned}
$$

We treat only the case where the first $n$ eigenvalues of $D$ are identical

$$
\delta_{1}=\delta_{2}=\cdots=\delta_{n} \equiv \delta
$$

and all other eigenvalues are different. All remaining cases are obvious generalizations.

The matrix $M$ is chosen as

$$
M=\left(\begin{array}{cc}
M_{0} & 0 \\
0 & \mathbb{1}_{N-n}
\end{array}\right),
$$

where $M_{0}$ is an $n \times n$ matrix that diagonalizes the submatrix

$$
B_{\text {sub }}=\left(\begin{array}{ccc}
B_{11} & \cdots & B_{1 n} \\
\vdots & & \vdots \\
B_{n 1} & \cdots & B_{n n}
\end{array}\right)
$$

of $B$, i.e.,

$$
M_{0}^{-1} B M_{0}=\left(\begin{array}{ccc}
\beta_{1} & & 0 \\
& \ddots & \\
0 & & \beta_{m}
\end{array}\right) .
$$

Because for the case considered

$$
D=\left(\begin{array}{cccc}
\delta 1 & & 0 & \\
& \delta_{n+1} & & 0 \\
0 & & \ddots & \\
& 0 & & \delta_{N}
\end{array}\right]
$$

the matrix $M$ obeys (A9). Moreover if we write

$$
B=\left(\begin{array}{cc}
B_{\text {sub }} & B_{1} \\
B_{2} & B_{3}
\end{array}\right)
$$

we obtain

$\widetilde{B} \equiv M^{-1} B M^{-1}=\left(\begin{array}{cccc}\beta_{1} & & 0 & \\ & \ddots & & M_{0}^{-1} B_{1} \\ 0 & & \beta_{n} & \\ & B_{2} M_{0} & & B_{3}\end{array}\right)$.

Entering this into (A10) gives in components 


$$
\left(\delta_{k}-\delta_{j}\right) L_{k j}+\widetilde{B}_{k j}=\kappa_{k} \delta_{k j}
$$

The solutions to these equations are

$$
L_{k j}=\frac{\widetilde{B}_{k j}}{\delta_{j}-\delta_{k}} \text { for } j \leq n<k \text { or } k \leq n<j
$$

and $L_{k j}$ otherwise arbitrary. Hence we have found a coordinate transformation that diagonalizes $A$ up to or$\operatorname{der} \epsilon$. The diagonal elements of (A18) reveal that the eigenvalues of $D_{1}$ are given by

$$
\kappa_{k}=\left\{\begin{array}{l}
\widetilde{B}_{k k}=\beta_{k} \text { for } k \leq n \\
\left(B_{3}\right)_{k k}=B_{k k} \text { for } k>n .
\end{array}\right.
$$

So in agreement with our proposition in the present case the eigenvalues of $A$ are given by

$$
\alpha_{k}=\left\{\begin{array}{l}
\delta+\epsilon \beta_{k}+O\left(\epsilon^{2}\right) \text { for } k \leq n \\
\delta+\epsilon B_{k k}+O\left(\epsilon^{2}\right) \text { for } k>n .
\end{array}\right.
$$

Along the same lines the proposition can be proved for other combinations of sets of degenerate and nondegenerate eigenvalues.

Proposition 2. If $A$ and $B$ are diagonalizable $n \times n$ matrices and $D$ a diagonal $n \times n$ matrix that obey

$$
A B=B A=D,
$$

then $A$ and $B$ can be simultaneously diagonalized by a permutation of indices.

Proof. According to (A22) $A$ and $B$ commute. Therefore they have a common set of eigenvectors with

$$
A v_{k}=\alpha_{k} v_{k} \text { and } B v_{k}=\beta_{k} v_{k} \text {. }
$$

$D$ is diagonal

[1] H. G. Schuster, Deterministic Chaos (Physik-Verlag, Weinheim, 1984).

[2] R. Van Buskirk and C. Jeffries, Phys. Rev. A 31, 3332 (1985).

[3] V. S. Anishchenko, Dynamical Chaos in Physical Systems (Teubner, Leipzig, 1989).

[4] J. Frøyland, Physica D 8, 423 (1983).

[5] M. P. Paulus, S. F. Gass, and A. J. Mandell, Physica D 40, 135 (1989).

[6] T. Hogg and B. A. Hubermann, Phys. Rev. A 29, 275 (1984); G. Mayer-Kress and G. Haubs, ibid. 30, 1127 (1984).

[7] M. F. Crowley and R. J. Field, J. Phys. Chem. 90, 1907 (1986).

[8] M. F. Crowley and I. R. Epstein, J. Phys. Chem. 93, 2496 (1989).

[9] R. H. Simoyi, A. Wolf, and H. L. Swinney, Phys. Rev. Lett. 49, 245 (1982).

[10] J. Wang, P. G. Sørensen, and F. Hynne, J. Phys. Chem. 98, 725 (1994).

[11] T. Geest, C. G. Steinmetz, R. Larter, and L. F. Olsen, J. Phys. Chem. 96, 5678 (1992).

[12] C. Knudsen, R. Feldberg, and H. True, Philos. Trans. R. Soc. London Ser. A 338, 455 (1992).

[13] R. S. Shaw, The Dripping Faucet as a Model Chaotic System (Aerial, Santa Cruz, 1984); P. Martien, S. C. Pope, P. L. Scott, and R. S. Shaw, Phys. Rev. Lett. 110A, 399

$$
D=\left(\begin{array}{lll}
\gamma_{1} & & 0 \\
& \ddots & \\
0 & & \gamma_{n}
\end{array}\right)
$$

and thus has the eigenvectors

$$
e_{k}=(0, \ldots, 1, \ldots, 0)^{T},
$$

where the entry " 1 " is in the $k$ th row. Let

$$
K \equiv\left(v_{1}, \ldots, v_{n}\right) \text {. }
$$

Then $K$ simultaneously diagonalizes $A$ and $B$ :

$$
\begin{aligned}
& K^{-1} A K=\left[\begin{array}{lll}
\alpha_{1} & & 0 \\
& \ddots & \\
0 & & \alpha_{n}
\end{array}\right], \\
& K^{-1} B K=\left(\begin{array}{llc}
\beta_{1} & & 0 \\
& \ddots & \\
0 & & \beta_{n}
\end{array}\right] .
\end{aligned}
$$

But

$$
D v_{k}=A B v_{k}=\alpha_{k} \beta_{k} v_{k} .
$$

Therefore $v_{k}$ is also an eigenvector of $D$ and thus there is a permutation $p:\{1 \cdots n\} \rightarrow\{1 \cdots n\}$ so that

$$
v_{k}=e_{p(k)}
$$

Therefore the transformation $K$ is equivalent to a permutation so that with (A27) and (A28) $A$ and $B$ are diagonal up to a permutation of indices.

(1985).

[14] K. S. Jensen, E. Mosekilde, and N.-H. Holstein-Rathlou, Mondes Dev. 54-55, 91 (1986); K. S. Jensen, N.-H. Holstein-Rathlou, P. P. Leyssac, E. Mosekilde, and D. R. Rasmussen, in Life Sciences: Chaos in Biological Systems, edited by H. Degn, A. V. Holden, and L. F. Olsen (Plenum, New York, 1987), p. 23.

[15] See, e.g., M. J. Hasler, Proc. IEEE 75, 1009 (1987).

[16] D. Ruelle, Trans. N.Y. Acad. Sci. 35, 66 (1973).

[17] J. C. Neu, SIAM J. Appl. Math. 37, 307 (1979).

[18] A. Klič, Apl. Mat. 31, 27 (1986).

[19] M. Kawato and R. Suzuki, J. Theor. Biol. 86, 547 (1980).

[20] L. Bauer, H. B. Keller, and E. L. Reiss, SIAM Rev. 17, 101 (1975).

[21] M. Golubitsky, I. Stewart, and D. G. Schaeffer, Singularities and Groups in Bifurcation Theory (Springer, New York, 1988), Vol. II.

[22] A. Klič, Apl. Mat. 28, 335 (1983).

[23] D. G. Aronson, E. J. Doedel, and H. G. Othmer, Physica D 25, 20 (1987).

[24] X.-J. Wang and G. Nicolis, Physica D 26, 140 (1987).

[25] D. G. Aronson, G. B. Ermentrout, and N. Kopell, Physica D 41, 403 (1990).

[26] M. A. Taylor and I. G. Kevrekides, Physica D 51, 274 (1991).

[27] K. Kaneko, Prog. Theor. Phys. 69, 1427 (1983).

[28] J.-M. Yuan, M. Tung, D. H. Feng, and L. M. Narducci, 
Phys. Rev. A 28, 1662 (1983).

[29] S. Kuznetsov, Radiophys. Quantum Electron. 28, 681 (1985).

[30] H. Fujisaka and T. Yamada, Prog. Theor. Phys. 69, 32 (1983).

[31] S.-Y. Kim, and H. Kook, Phys. Rev. A 46, R4467 (1992).

[32] I. Schreiber and M. Marek, Phys. Lett. 91, 263 (1982).
[33] M. Marek and I. Schreiber, Chaotic Behaviour of Deterministic Dissipative Systems (Cambridge University Press, Cambridge, England, 1991).

[34] S.-N. Chow and J. Hale, Methods of Bifurcation Theory (Springer, Berlin, 1982).

[35] D. Ruelle, Arch. Ration. Mech. Anal. 51, 136 (1973).

[36] S.-Y. Kim and H. Kook, Phys. Rev. E 48, 785 (1993). 